Review

\title{
Emerging Biomarkers in Vascular Cognitive Impairment and Dementia: From Pathophysiological Pathways to Clinical Application
}

\author{
Virginia Cipollini *, Fernanda Troili and Franco Giubilei \\ S. Andrea Hospital, NESMOS Department, Faculty of Medicine and Psychology, Sapienza University of Rome, \\ Via di Grottarossa 1035, 00189 Roma, Italy; f.troili86@gmail.com (F.T.); franco.giubilei@uniroma1.it (F.G.) \\ * Correspondence: virginia.cipollini@gmail.com; Tel.: +39-3494216157
}

Received: 26 April 2019; Accepted: 3 June 2019; Published: 8 June 2019

\begin{abstract}
Vascular pathology is the second most common neuropathology of dementia after Alzheimer's disease (AD), with small vessels disease (SVD) being considered the major cause of vascular cognitive impairment and dementia (VCID). This review aims to evaluate pathophysiological pathways underlying a diagnosis of VCID. Firstly, we will discuss the role of endothelial dysfunction, blood-brain barrier disruption and neuroinflammation in its pathogenesis. Then, we will analyse different biomarkers including the ones of inflammatory responses to central nervous system tissue injuries, of coagulation and thrombosis and of circulating microRNA. Evidences on peripheral biomarkers for VCID are still poor and large-scale, prospectively designed studies are needed to translate these findings into clinical practice, in order to set different combinations of biomarkers to use for differential diagnosis among types of dementia.
\end{abstract}

Keywords: vascular disease; vascular dementia; vascular cognitive impairment; peripheral biomarkers; blood biomarkers; CSF biomarkers

\section{Background}

Vascular Cognitive Impairment and Dementia: An Umbrella Term

In recent years, many efforts have been made to achieve consensus in defining cognitive impairment with vascular pathology [1] and the term vascular cognitive impairment and dementia (VCID) is recently being used as an umbrella term [2,3]. Despite the variable prevalence estimated in the literature, varying according to the employed diagnostic criteria, VCID is recognized as the second most common cause of dementia after Alzheimer's disease (AD) [4]. The term VCID refers to a group of different diseases characterized by cognitive impairment caused primarily by cerebrovascular disease (CVD). This includes the full spectrum ranging from vascular mild cognitive impairment (MCI) to Vascular Dementia (VaD) [4]. Recently, due to this heterogeneity, the Society for Vascular Behavioral and Cognitive Disorders (VASCOG) produced criteria for vascular cognitive disorder [5] which were harmonized with the DSM-5 criteria [6]. Core features include: stepwise progression, focal neurological signs and symptoms, unequal distribution of cognitive deficits, history of multiple ischemic strokes, neuroimaging evidence of cerebrovascular disease and temporal relationship between CVD and cognitive impairment [7].

Nowadays, there is a strong scientific rationale for linking CVD to cognitive impairment, including the link between age-related cerebrovascular changes and dysregulation of cerebral perfusion, blood-brain barrier (BBB) function, and neurovascular unit (NVU) coupling. The range of neuropathological mechanisms underlying a diagnosis of VCID include: post-stroke impairment, 
small and large vessel diseases, and cases of mixed-pathology, with CVD that interacts with AD pathology [8].

Initially, vascular dementia was thought to be related to the volume of multiple strokes, condition called multi-infarct dementia (MID) [9]. Then, the use of brain imaging revealed a high incidence of white matter damage not necessarily related to strokes. In this regard, there was a growing awareness of the importance of slowly progressive changes in the brain related to small vessels disease (SVD) [10]. It is now thought by many investigators that SVD is the major cause of VCID and the most common pathology found in the elderly, doubling the chances that neurodegenerative pathology would lead to dementia [11].

In the past years, research on biomarkers has mainly focused to relate candidate markers to specific neuroimaging features, such as lacunar infarcts and white matter hyperintensities (WMHs) in population-based studies or in patients with a high risk of vascular factors. Other studies have compared possible biomarkers according to the different subtypes of VCID disorders. Indeed, advances in neuroimaging and biochemical analysis of blood and cerebrospinal fluid (CSF) provide unique multimodal biomarkers to stratify VCID patients [12,13].

In this review, we will briefly summarize the evidences on emerging blood and CSF biomarkers related to VCID, particularly with SVD, a common, fairly homogeneous, but often under-recognized histopathologic subtype of VCID. Firstly, we will consider the pathophysiological pathways underlying a diagnosis of VCID and we will discuss the role of endothelial dysfunction, blood-brain barrier disruption and neuroinflammation in its pathogenesis. Then we will analyse different biomarkers including biomarkers of inflammatory responses, biomarkers of central nervous system tissue injuries, biomarkers of coagulation and thrombosis, and circulating microRNA (miRNA).

\section{Methods}

For this narrative review, we conducted a comprehensive search on Medline and PubMed databases for studies published until 31st March 2019. The keywords used in the current search were: "vascular dementia" or "vascular cognitive impairment" or "subcortical small vessel disease" AND "biomarkers", "inflammation", "blood brain barrier", "endothelial dysfunction", "microRNA". We used filters for English language and selected manuscripts not older than 10 years. Two of the authors, VC and FT, separately selected relevant abstracts and critically reviewed them. The full text of 197 relevant papers were taken into account. Moreover, 24 reviews from the last 5 years that could be of interest for our work were considered. The proposal on current status of biochemical markers in VCID was reviewed by the group.

For the tables compilation filters for publication dates not older than the last 5 years, only for human subjects and only in English language were applied, obtaining 326 articles to analyse. We then excluded all the reviews and not pertinent topics by reading article abstracts, and then obtained all the salient full texts (49 articles in total) (Tables 1 and 2).

\section{Pathophysiological Pathways of VCID}

The heterogeneity of CVD makes it challenging to elucidate the neuropathological substrates and mechanisms of VCID. VCID is an entity whose heterogeneous clinical manifestations are due to a substrate of multiple pathogenic and structural factors. Nevertheless, histopathologic evidence, obtained by biopsy or autopsy, is essential in each guideline to make a diagnosis of definite VCID [14]. Decreased Cerebral Blood Flow (CBF) is the major cerebral hemodynamic alteration in VCID and pathologies, which cause reduction in global CBF, such as atherosclerosis and arterial stenosis are involved [15]. Factors that define the subtypes of VCID include the nature and extent of vascular pathologies (such as ischemic infarcts, hemorrhages and white matter changes), the degree of involvement of extra and intracranial vessels and the anatomical location of tissue changes. Typical neuropathological changes of AD such as amyloid plaques and neurofibrillary tangles may also be found at the pathological examination of VCID and may contribute to cognitive dysfunction $[5,16]$. 
Table 1. Blood biomarkers and VCID.

\begin{tabular}{|c|c|c|c|c|}
\hline $\begin{array}{l}\text { Author, Year } \\
\text { [Reference] }\end{array}$ & Population & Biomakers & Outcome & Results \\
\hline $\begin{array}{l}\text { Murr J et al., } 2014 \\
\text { [17] }\end{array}$ & 670 cognitively normal subjects & $\begin{array}{l}\text { Plasma oxidized low-density } \\
\text { lipoprotein (OxLDL) levels }\end{array}$ & $\begin{array}{c}\text { Risk Factor } \\
\text { follow-up: } 4.8 \text { years }(2.2-10.3)\end{array}$ & $\begin{array}{l}\text { No association between OxLDL and risk of } \\
\text { all-cause dementia, AD, and vascular } \\
\text { dementia or subtypes was found. }\end{array}$ \\
\hline $\begin{array}{c}\text { Dong H et al., } 2015 \\
{[18]}\end{array}$ & $\begin{array}{l}127 \mathrm{AD} \text { patients, } 30 \mathrm{MCI} \text { and } 30 \mathrm{VaD} \\
\text { patients. }\end{array}$ & Serum circulating miRNAs & Differential diagnosis & $\begin{array}{l}\text { The panel of miR-31, miR-93 and miR-146a } \\
\text { can be used to discriminate AD from VaD. }\end{array}$ \\
\hline $\begin{array}{c}\text { Duits FH et al., } 2015 \\
\text { [19] }\end{array}$ & $\begin{array}{l}52 \text { AD patients, } 24 \mathrm{VaD} \text { patients, } 26 \\
\text { cognitively normal subjects. }\end{array}$ & $\begin{array}{c}\text { Plasma and CSF levels of MMP2, } \\
\text { MMP9, MMP10, TIMP1 and TIMP2. } \\
\text { CSF levels of amyloid- } \beta(1-42) \\
\text { (A } \beta 42) \text {, tau, and tau } \\
\text { phosphorylated at threonine-181 } \\
\text { (p-tau). }\end{array}$ & Differential diagnosis & $\begin{array}{l}\text { AD patients showed higher plasma MMP2 } \\
\text { levels compared to VaD patients }(p<0.05)\end{array}$ \\
\hline $\begin{array}{c}\text { Hatanaka H et al., } 2015 \\
\text { [20] }\end{array}$ & $\begin{array}{c}72 \mathrm{AD}, 27 \mathrm{VaD}, 24 \text { Mixed Dementia } \\
\text { (MD) patients and } 53 \text { cognitively } \\
\text { normal subjects. }\end{array}$ & $\begin{array}{l}\text { Plasma levels of diacron reactive } \\
\text { oxygen metabolite (dROM) and } \\
\text { biological anti-oxidant potential } \\
\text { (BAP). }\end{array}$ & Differential diagnosis & $\begin{array}{l}\text { The dROM levels were significantly higher } \\
\text { in the AD and MD groups than in the } \\
\text { control group. } \\
\text { The BAP levels were significantly lower in } \\
\text { the MD group than in the control, AD and } \\
\text { VaD groups. }\end{array}$ \\
\hline $\begin{array}{l}\text { Hilal S et al., } 2015 \\
{[21]}\end{array}$ & $\begin{array}{c}41 \text { cognitive impairment with no } \\
\text { dementia (CIND) subjects and } 46 \\
\text { demented subjects with burden of } \\
\text { cerebrovascular diseases (CeVD); } 37 \\
\text { CIND and } 34 \text { cases with dementia } \\
\text { without CeVD; } 35 \text { cognitively normal } \\
\text { subjects. }\end{array}$ & $\begin{array}{l}\text { Plasma NTpro-BNP and high } \\
\text { sensitivity cardiac troponin T } \\
\text { (hs-cTnT) levels. }\end{array}$ & Differential diagnosis & $\begin{array}{l}\text { Plasma concentrations of hs-cTnT were } \\
\text { associated significantly with CeVD in both } \\
\text { CIND and dementia. } \\
\text { NTpro-BNP was associated with dementia } \\
\text { with CeVD }\end{array}$ \\
\hline $\begin{array}{c}\text { Teunissen CE et al., } 2015 \\
\text { [22] }\end{array}$ & $\begin{array}{l}295 \text { subjects including healthy controls } \\
(n=65) \text {, patients with subjective } \\
\text { memory complaints }(n=99) \text {, patients } \\
\text { with } \mathrm{AD}(n=100) \text {, and patients with } \\
\text { vascular dementia }(n=31) .\end{array}$ & Serum leptin levels & Differential diagnosis & $\begin{array}{l}\text { Serum leptin levels are not altered in AD or } \\
\text { vascular dementia patients compared to } \\
\text { healthy controls and were not related to } \\
\text { cognitive decline. }\end{array}$ \\
\hline
\end{tabular}


Table 1. Cont.

\begin{tabular}{|c|c|c|c|c|}
\hline $\begin{array}{l}\text { Author, Year } \\
\text { [Reference] }\end{array}$ & Population & Biomakers & Outcome & Results \\
\hline $\begin{array}{l}\text { Wang C et al., } 2015 \\
\text { [23] }\end{array}$ & $\begin{array}{l}10 \text { cognitively healthy subjects and } 10 \\
\text { age-matched VaD subjects. }\end{array}$ & $\begin{array}{l}\text { Differentially expressed proteins } \\
\text { (DEPs) }\end{array}$ & Differential diagnosis & $\begin{array}{c}\text { High-degree proteins were detected in the } \\
\text { protein-protein (PPI) interaction network, } \\
\text { such as ATP5B (ATP synthase subunit } \beta \text { ) in } \\
\text { VaD. }\end{array}$ \\
\hline $\begin{array}{c}\text { Castellazzi M et al., } 2016 \\
\text { [24] }\end{array}$ & $\begin{array}{l}232 \mathrm{MCI}, 65 \mathrm{VaD}, 175 \mathrm{AD}, 88 \mathrm{MD}, 104 \\
\text { Multiple Sclerosis (MS) patients, } 165 \\
\text { cognitively healthy controls. }\end{array}$ & $\begin{array}{l}\text { Serum High-density lipoprotein } \\
\text { (HDL)-bound paraoxonase-1 } \\
\text { (PON-1) levels }\end{array}$ & Differential diagnosis & $\begin{array}{c}\text { Serum arylesterase, but not paraoxonase, } \\
\text { levels were significantly lower in patients } \\
\text { affected by MCI, VaD, AD, MD as well as } \\
\text { those with MS as compared to healthy } \\
\text { controls. }\end{array}$ \\
\hline $\begin{array}{l}\text { Chen Z et al., } 2016 \\
\text { [25] }\end{array}$ & $\begin{array}{l}52 \text { subcortical ischemic vascular disease } \\
\text { patients with no dementia (SIVDND), } \\
55 \text { patients with mild cognitive } \\
\text { impairment (SVMCI), } 54 \text { patients with } \\
\text { vascular dementia (VaD), } 54 \text { cognitively } \\
\text { healthy controls. }\end{array}$ & $\begin{array}{c}\text { Serum thyroid-stimulating } \\
\text { hormone (TSH), total } \\
\text { triiodothyronine (TT3), free } \\
\text { triiodothyronine (FT3), total } \\
\text { thyroxine (TT4) and free thyroxine } \\
\text { (FT4), thyroglobulin antibody } \\
\text { (TGA), and antithyroid peroxidase } \\
\text { antibody (TPO-Abs). }\end{array}$ & $\begin{array}{l}\text { Correlation with cognitive } \\
\text { status }\end{array}$ & $\begin{array}{l}\text { Serum TT3 and FT3 levels decreased, } \\
\text { whereas serum TSH level increased, with } \\
\text { the decline in cognitive functions in SIVD. }\end{array}$ \\
\hline $\begin{array}{l}\text { Dukic L et al., } 2016 \\
\text { [26] }\end{array}$ & $\begin{array}{c}235 \text { participants, divided in } 4 \text { groups: } \\
\mathrm{AD}(N=70), \mathrm{VaD}(N=67), \mathrm{MCI}(N= \\
48) \text { and Cognitively Healthy } \\
\text { Participants }(N=50)\end{array}$ & $\begin{array}{c}\text { Serum kallikrein } 6 \text { (KLK6), clusterin } \\
\text { (CLU), adiponectin (ADPN) and } \\
\text { interleukin-6 (IL-6) }\end{array}$ & Differential diagnosis & $\begin{array}{l}\text { Serum concentrations of KLK6, CLU and } \\
\text { ADPN did not differ between AD, VaD, } \\
\text { MCI and cognitively healthy control group } \\
\text { of participants, whereas IL- } 6 \text { was } \\
\text { significantly higher in VaD patients than in } \\
\text { AD, MCI and healthy individuals. }\end{array}$ \\
\hline $\begin{array}{c}\text { Horvath I et al., } 2016 \\
\text { [27] }\end{array}$ & $\begin{array}{c}40 \text { nondemented controls, } 11 \text { stable } \\
\text { mild cognitive impairment (SMCI), } 6 \\
\text { MCI due to AD (MCI-AD), } 40 \text { AD and } 7 \\
\text { VaD.patients. }\end{array}$ & $\begin{array}{l}\text { CSF concentration of } A \beta 1-42, \\
\text { S100A8, S100A9 and Tau. }\end{array}$ & Differential diagnosis & $\begin{array}{l}\text { The S100A9 and A } \beta 1-42 \text { levels correlated } \\
\text { with each other: their CSF content } \\
\text { decreased already at the SMCI stage and } \\
\text { declined further under MCIAD, AD, and } \\
\text { VaD conditions. }\end{array}$ \\
\hline
\end{tabular}


Table 1. Cont

\begin{tabular}{|c|c|c|c|c|}
\hline $\begin{array}{l}\text { Author, Year } \\
\text { [Reference] }\end{array}$ & Population & Biomakers & Outcome & Results \\
\hline $\begin{array}{c}\text { Kitagawa K et al., } 2016 \\
\text { [28] }\end{array}$ & 466 cognitively healthy subjects & $\begin{array}{l}\text { serum high-molecular-weight } \\
\text { (HMW) adiponectin level }\end{array}$ & $\begin{array}{l}\text { Risk factor (median follow-up } \\
\text { period: } 6.9 \text { years) }\end{array}$ & $\begin{array}{l}\text { Risks of dementia in patients with high } \\
\text { versus low HMW adiponectin levels were } \\
\text { almost identical. } \\
\text { No association was found between } \\
\text { adiponectin levels and Alzheimer's disease } \\
\text { dementia or vascular dementia in the } \\
\text { whole group or amongst men and women } \\
\text { separately. }\end{array}$ \\
\hline $\begin{array}{l}\text { Levada OA et al., } 2016 \\
\text { [29] }\end{array}$ & $\begin{array}{l}21 \text { patients with } \mathrm{AD} ; 22 \text { patients with } \\
\text { subcortical vascular dementia; } 16 \\
\text { cognitively healthy subjects. }\end{array}$ & $\begin{array}{l}\text { Plasma levels of brain-derived } \\
\text { neurotrophic factor (BDNF) }\end{array}$ & Differential diagnosis & $\begin{array}{l}\text { At baseline there was lower BDNF levels in } \\
\text { both AD and VaD groups, which was } \\
\text { significant only in subjects with AD. }\end{array}$ \\
\hline $\begin{array}{c}\text { Mirza SS et al., } 2016 \\
{[30]}\end{array}$ & $357 \mathrm{AD}$ patients; $32 \mathrm{VaD}$ patients. & $\begin{array}{l}\text { Serum N-terminal pro B-type } \\
\text { natriuretic peptide (NT-proBNP) } \\
\text { levels }\end{array}$ & Risk factor & $\begin{array}{l}\text { Higher NT-proBNP was associated with a } \\
\text { higher risk of dementia, with a particularly } \\
\text { strong association with vascular dementia. }\end{array}$ \\
\hline $\begin{array}{l}\text { Nilsson ED et al., } 2016 \\
\text { [31] }\end{array}$ & $\begin{array}{l}374 \text { incident dementia cases: } 120 \mathrm{AD} \\
\text { patients, } 84 \mathrm{VaD} \text { and } 102 \mathrm{MD} \text { patients. }\end{array}$ & Plasma level of copeptin & $\begin{array}{l}\text { Risk factor (median follow-up } \\
\text { period: } 4.2 \text { years) }\end{array}$ & $\begin{array}{l}\text { Baseline level of copeptin predicted } \\
\text { incident VaD. }\end{array}$ \\
\hline $\begin{array}{c}\text { Pan X et al., } 2016 \\
{[32]}\end{array}$ & $\begin{array}{l}\text { Exploration phase: } 338 \text { control subjects } \\
\text { and } 43 \text { AD patients; } \\
\text { Validation phase: } 861 \text { control subjects, } \\
81 \text { AD patients, } 70 \text { vascular dementia } \\
\text { patients and } 13 \text { frontotemporal } \\
\text { dementia patients. }\end{array}$ & $\begin{array}{l}\text { Blood Thiamine diphosphate (TDP), } \\
\text { thiamine monophosphate, and } \\
\text { thiamine levels }\end{array}$ & Differential diagnosis & $\begin{array}{l}\text { TDP exhibited significant and consistent } \\
\text { values for AD diagnosis in both } \\
\text { exploration and validation phases. } \\
\text { TDP can effectively distinguish AD from } \\
\text { vascular dementia. }\end{array}$ \\
\hline $\begin{array}{l}\text { Bednarska-Makaruk M } \\
\text { et al., } 2017 \\
\text { [33] }\end{array}$ & $\begin{array}{l}205 \text { patients with dementia ( } 89 \text { with } \\
\text { AD, } 47 \text { with VaD, } 69 \text { with mixed } \\
\text { dementia (MD)), } 113 \text { persons with MCI } \\
\text { and } 107 \text { controls. }\end{array}$ & $\begin{array}{l}\text { Serum adiponectin, leptin and } \\
\text { resistin levels, IL-6, CRP, } \\
\text { chitotriosidase, 25-OH vitamin D, } \\
\text { HDL-cholesterol and paraoxonase 1, } \\
\text { glucose, insulin and HOMA-IR. }\end{array}$ & Differential diagnosis & $\begin{array}{l}\text { Vascular and mixed dementia are } \\
\text { characterized by an increase of resistin. } \\
\text { Positive correlation of resistin with } \\
\text { inflammation indicators may suggest the } \\
\text { potential pro-inflammatory role of resistin } \\
\text { in the development of dementia, especially } \\
\text { in dementia of vascular mechanism. }\end{array}$ \\
\hline
\end{tabular}


Table 1. Cont.

\begin{tabular}{|c|c|c|c|c|}
\hline $\begin{array}{l}\text { Author, Year } \\
\text { [Reference] }\end{array}$ & Population & Biomakers & Outcome & Results \\
\hline $\begin{array}{l}\text { Busse M et al., } 2017 \\
{[34]}\end{array}$ & $\begin{array}{l}60 \mathrm{AD} \text { patients, } 20 \mathrm{VaD} \text { patients, } 12 \\
\text { frontotemporal dementia patients and } \\
24 \text { cognitively healthy persons. }\end{array}$ & $\begin{array}{l}\text { Innate and adaptive cell } \\
\text { populations in whole blood. }\end{array}$ & Differential diagnosis & $\begin{array}{l}\text { Monocytes and NK cells were diminished } \\
\text { in VaD, but not in AD and FTD. } \\
\text { B cell and T cell numbers were decreased in } \\
\text { all investigated forms of dementia. } \\
\text { Changes in the contribution of } \\
\text { naïve/memory T cells were only present in } \\
\text { AD. }\end{array}$ \\
\hline $\begin{array}{l}\text { Holm H et al., } 2017 \\
{[35]}\end{array}$ & $\begin{array}{c}120 \mathrm{AD} \text { patients, } 83 \mathrm{VaD}, 102 \mathrm{MD} \text { and } \\
68 \text { other aetiology. }\end{array}$ & $\begin{array}{l}\text { Plasma N-terminal prosomatostatin } \\
\text { (NT-proSST) }\end{array}$ & $\begin{array}{c}\text { Risk factor } \\
\text { (follow-up period of } 4.6 \pm 1.3 \\
\text { years) }\end{array}$ & $\begin{array}{l}\text { Higher levels of circulating NTproSST are } \\
\text { associated with increased incidence of } \\
\text { vascular dementia. }\end{array}$ \\
\hline $\begin{array}{l}\text { Holm H et al., } 2017 \\
{[36]}\end{array}$ & $\begin{array}{l}\text { A population-based cohort of } 5347 \\
\text { individuals without prevalent dementia }\end{array}$ & $\begin{array}{c}\text { Plasma midregional pro-atrial } \\
\text { natriuretic peptide (MR-proANP), } \\
\text { C-terminal endothelin-1 } \\
\text { (CT-proET-1) and midregional } \\
\text { proadrenomedullin (MR-proADM). }\end{array}$ & $\begin{array}{l}\text { Risk factor (follow-up period of } \\
\qquad 4.6 \pm 1.3 \text { years) }\end{array}$ & $\begin{array}{l}\text { Elevated plasma concentration of } \\
\text { MR-proANP is an independent predictor } \\
\text { of all-cause and vascular dementia. } \\
\text { Pronounced increase in CT-proET-1 } \\
\text { indicates higher risk of vascular dementia. }\end{array}$ \\
\hline $\begin{array}{c}\text { Hsu PF et al., } 2017 \\
\text { [37] }\end{array}$ & $\begin{array}{l}1436 \text { individuals from a national } \\
\text { representative sample in Taiwan. }\end{array}$ & CRP levels were determined. & $\begin{array}{l}\text { Risk Factor (11.04 years } \\
\text { (median) of follow-up) }\end{array}$ & $\begin{array}{l}260 \text { individuals }(18.11 \%) \text { were diagnosed } \\
\text { with dementia. Those with high CRP had a } \\
55 \% \text { higher risk of dementia compared with } \\
\text { those with normal CRP. After adjusting for } \\
\text { possible confounding cardiovascular risk } \\
\text { factors, high CRP was independently } \\
\text { associated with VaD, but not AD. }\end{array}$ \\
\hline
\end{tabular}


Table 1. Cont

\begin{tabular}{|c|c|c|c|c|}
\hline $\begin{array}{l}\text { Author, Year } \\
\text { [Reference] }\end{array}$ & Population & Biomakers & Outcome & Results \\
\hline $\begin{array}{c}\text { Moretti R et al., } 2017 \\
\text { [38] }\end{array}$ & $\begin{array}{l}543 \text { patients: } 456 \text { patients suffering } \\
\text { from subcortical vascular dementia } \\
\text { (sVAD), } 87 \text { AD and healthy } \\
\text { age-matched controls }\end{array}$ & $\begin{array}{l}\text { Clinical laboratory measurements, } \\
\text { including serum total cholesterol, } \\
\text { triglycerides, and high-density } \\
\text { lipoprotein (HDL) cholesterol, have } \\
\text { been determined enzymatically and } \\
\text { low-density lipoprotein (LDL) } \\
\text { cholesterol was calculated using } \\
\text { Friedewald's formula. Serum levels } \\
\text { of } 25(\mathrm{OH}) \mathrm{D} \text {, the level of calcium } \\
\text { and PTH were measured.The level } \\
\text { of folate, vitamin B12 levels and } \\
\text { Homocysteine were also tested. }\end{array}$ & Correlation & $\begin{array}{l}\text { Vitamin D deficiency was present in } \\
\text { demented cases, as well as low levels of } \\
\text { folate and high levels of homocysteine, } \\
\text { more pronounced in sVAD cases. }\end{array}$ \\
\hline $\begin{array}{c}\text { Prabhakar P et al., } 2017 \\
\text { [39] }\end{array}$ & $\begin{array}{l}204 \text { patients with small vessel VaD; } 200 \\
\text { cognitively normal subjects. }\end{array}$ & Plasma miRNA profiling. & Differential diagnosis & $\begin{array}{l}\text { plasma miR-409-3p, miR-502-3p, } \\
\text { miR-486-5p and miR-451a could be used to } \\
\text { differentiate small vessel VaD patients from } \\
\text { healthy controls. }\end{array}$ \\
\hline $\begin{array}{l}\text { Quinlan P et al., } 2017 \\
\text { [40] }\end{array}$ & $\begin{array}{l}342 \text { patients with subjective or objective } \\
\text { mild cognitive impairment }\end{array}$ & $\begin{array}{l}\text { Serum IGF-I concentrations at } \\
\text { baseline }\end{array}$ & $\begin{array}{l}\text { Risk factor (mean follow-up: } 3.6 \\
\text { years) }\end{array}$ & $\begin{array}{l}\text { In a memory clinic population, low serum } \\
\text { IGF-I was a risk marker for subsequent } \\
\text { VaD whereas low IGF-I did not associate } \\
\text { with the risk of AD. High serum IGF-I was } \\
\text { not related to the risk of conversion to } \\
\text { dementia. }\end{array}$ \\
\hline $\begin{array}{c}\text { Suridjan I et al., } 2017 \\
\text { [41] }\end{array}$ & $\begin{array}{l}29 \text { possible vascular mild cognitive } \\
\text { impairment patients and } 89 \text { controls) }\end{array}$ & $\begin{array}{l}\text { Serum lipid peroxidation markers } \\
\text { levels. } \\
\text { Ratios of early- (lipid } \\
\text { hydroperoxides, LPH) to late-stage } \\
\text { (8-isoprostane, 8-ISO; } \\
\text { 4-hydroxy-2-nonenal, 4-HNE) lipid } \\
\text { peroxidation products were } \\
\text { calculated. }\end{array}$ & $\begin{array}{l}\text { Correlation with cognitive } \\
\text { status }\end{array}$ & $\begin{array}{c}\text { A global effect of group on lipid } \\
\text { peroxidation markers was observed, } \\
\text { adjusting for sex, years of education, and } \\
\text { cardiopulmonary fitness. } \\
\text { Lower lipid peroxidation at baseline, as } \\
\text { determined by lower 8-ISO concentration, } \\
\text { was associated with greater improvement } \\
\text { in verbal memory }(F(1,64)=4.738, p= \\
0.03) \text { and executive function }(F(1,64)= \\
5.219, p=0.026) \text { performance. } \\
\text { Higher ratios of 8-ISO/LPH and } \\
\text { 8-ISO+4-HNE to LPH, were associated } \\
\text { with less improvement in executive } \\
\text { function performance over a 24-week } \\
\text { exercise intervention. }\end{array}$ \\
\hline
\end{tabular}


Table 1. Cont.

\begin{tabular}{|c|c|c|c|c|}
\hline $\begin{array}{l}\text { Author, Year } \\
\text { [Reference] }\end{array}$ & Population & Biomakers & Outcome & Results \\
\hline $\begin{array}{c}\text { Tang SC et al., } 2017 \\
{[42]}\end{array}$ & $\begin{array}{c}172 \text { Ischemic Stroke (IS) patients, } \\
\text { including } 73 \text { with } C D R=0,63 \text { with } \\
\mathrm{CDR}=0.5 \text {, and } 36 \text { with } \mathrm{CDR} \geq 1 \text { (VaD } \\
\text { patients). }\end{array}$ & $\begin{array}{c}\text { Plasma concentration of receptor } \\
\text { for advanced glycation end } \\
\text { products (soluble RAGE (sRAGE); } \\
\text { endogenous soluble form of RAGE } \\
\text { (esRAGE)) }\end{array}$ & $\begin{array}{l}\text { Correlation with cognitive } \\
\text { status }\end{array}$ & $\begin{array}{l}\text { Plasma sRAGE and esRAGE were elevated } \\
\text { in patients with dementia compared with } \\
\text { those without dementia among IS patients. }\end{array}$ \\
\hline $\begin{array}{l}\text { Vishnu VY et al., } 2017 \\
\text { [43] }\end{array}$ & $\begin{array}{l}118 \text { subects, } 68 \text { with dementia (MCI-AD } \\
\text { and AD: 52; MCI VaSC and VaD:16). }\end{array}$ & $\begin{array}{l}\text { Plasma IL-6; } \\
\text { C-Reactive Protein }(\mathrm{CRP}) ; \\
\text { plasma fibrinogen; } \\
\text { plasma D-dimer. }\end{array}$ & Differential diagnosis & $\begin{array}{l}\text { Plasma Fibrinogen and D dimer: were } \\
\text { higher in Vascular group; no difference in } \\
\text { IL-6 and CRP. }\end{array}$ \\
\hline $\begin{array}{l}\text { Wang R et al.,2017 } \\
\text { [44] }\end{array}$ & $\begin{array}{l}88 \text { patients with dementia ( } 43 \mathrm{AD} \\
\text { patients, } 45 \mathrm{VaD} \text { patients) and } 45 \\
\text { healthy age-matched controls }\end{array}$ & $\begin{array}{c}\text { Plasma Cystatin C (Cys C) and } \\
\text { HDL levels }\end{array}$ & Differential diagnosis & $\begin{array}{l}\text { Plasma Cys } C \text { levels were higher in patients } \\
\text { with } \mathrm{AD} / \mathrm{VaD} \text { than in healthy subjects. } \\
\text { Plasma HDL levels were lower in patients } \\
\text { with } \mathrm{AD} / \mathrm{VaD} \text { than in healthy subjects. }\end{array}$ \\
\hline $\begin{array}{l}\text { Yang R et al., } 2017 \\
\text { [45] }\end{array}$ & $\begin{array}{l}51 \text { healthy subjects and } 41 \text { elderly } \\
\text { patients (with } 6 \text { participants affected by } \\
\text { vascular mild cognitive impairment } \\
\text { (Va-MCI)), } 9 \text { with VaD, } 8 \text { with MCI due } \\
\text { to AD and } 18 \text { with AD }\end{array}$ & Plasma GDF-11 and $\beta 2-$ MG levels & Differential diagnosis & $\begin{array}{l}\text { No differences in circulating GDF-11 levels } \\
\text { amongst the healthy advanced age and } \\
\text { four cognitive impairment groups. } \beta 2-\mathrm{MG} \\
\text { levels increased with age, but there was no } \\
\text { significant difference between healthy } \\
\text { elderly males and advanced age males. } \\
\text { Increased levels of } \beta 2-\mathrm{MG} \text { were observed } \\
\text { in the dementia group compared with the } \\
\text { healthy advanced age group. }\end{array}$ \\
\hline $\begin{array}{l}\text { Brombo G et al., } 2018 \\
\text { [46] }\end{array}$ & $\begin{array}{l}320 \text { elderly individuals ( } \geq 65 \text { years old): } \\
60 \text { patients with normal cognition; } 60 \\
\text { patients with VaD; } 100 \text { patients with } \\
\text { AD; } 100 \text { patients with } \mathrm{MCI} \text {. }\end{array}$ & Plasma Klotho levels & Differential diagnosis & $\begin{array}{l}\text { Lower levels of plasma Klotho (1st tertile) } \\
\text { were associated with higher prevalence of } \\
\text { VaD, but not AD. }\end{array}$ \\
\hline $\begin{array}{l}\text { Latourte A et al., } 2018 \\
\text { [47] }\end{array}$ & 1598 people. & Serum Uric Acid (SUA) & $\begin{array}{c}\text { Risk Factor } \\
\text { (median folow-up duration: } \\
10.1 \text { years) }\end{array}$ & $\begin{array}{c}\text { Association for development of dementia } \\
\text { was stronger with vascular or mixed } \\
\text { dementia (HR = 3.66 ( } 95 \% \text { CI } 1.29 \text { to } 10.41) \text {, } \\
p=0.015) \text { than Alzheimer's disease (HR = } \\
1.55 \text { ( } 95 \% \text { CI } 0.92 \text { to } 2.61), p=0.10) .\end{array}$ \\
\hline
\end{tabular}


Table 1. Cont.

\begin{tabular}{|c|c|c|c|c|}
\hline $\begin{array}{l}\text { Author, Year } \\
\text { [Reference] }\end{array}$ & Population & Biomakers & Outcome & Results \\
\hline $\begin{array}{l}\text { Lauriola M et al., } 2018 \\
\text { [48] }\end{array}$ & $\begin{array}{c}116 \text { patients: } 32 \text { healthy controls, } 39 \\
\text { with diagnosis of VaD, } 14 \text { MCI and } 31 \\
\text { AD. }\end{array}$ & $\begin{array}{l}\text { Erythrocyte associated } A \beta \text { (iA } \beta 40 \\
\text { and } i A \beta 42 \text { ) levels }\end{array}$ & Differential diagnosis & $\begin{array}{l}\text { AD showed different iA } \beta 42 \text { levels as } \\
\text { compared to VaD. Conversely, no } \\
\text { differences were found for iA } \beta 40 \text {. }\end{array}$ \\
\hline $\begin{array}{c}\text { Shang J et al., } 2018 \\
\text { [49] }\end{array}$ & $\begin{array}{l}266 \text { patients with } \mathrm{AD}, 44 \mathrm{MCI}, 33 \mathrm{VaD} \\
\text { and } 200 \text { ischemic stroke (IS) in } \\
\text { comparison to } 130 \text { healthy controls. }\end{array}$ & $\begin{array}{c}\text { Plasma fatty acids } \\
\text { [eicosapentaenoic acid (EPA) and } \\
\text { docosahexaenoic acid (DHA)], } \\
\text { adiponectin, reptin, plasma } \\
\text { markers of inflammation } \\
\text { [high-sensitivity C-reactive protein } \\
\text { (hsCRP) and serum amyloid A } \\
\text { (serum AA)], and plasma lipids } \\
\text { [high-density lipoprotein and } \\
\text { low-density lipoprotein (LDL)] }\end{array}$ & Differential disgnosis & $\begin{array}{c}\text { Lower EPA and DHA levels and higher } \\
\text { reptin and LDL levels were associated with } \\
\text { AD and IS. The reptin/adiponectin ratio } \\
\text { was strongly associated with IS. The hsCRP } \\
\text { level was more strongly associated with } \\
\text { VaD and IS, and the serum AA level was } \\
\text { associated with all three cognitive diseases } \\
\text { and IS. }\end{array}$ \\
\hline $\begin{array}{c}\text { Staszewski J et al., } 2018 \\
\text { [50] }\end{array}$ & $\begin{array}{l}123 \text { patients (age, mean } \pm \text { SD: } 72.2 \pm 8 \\
\text { years, } 49 \% \text { females), with lacunar stroke } \\
(n=49), \text { vascular dementia }(n=48), \\
\text { and vascular parkinsonism }(n=26) .\end{array}$ & $\begin{array}{l}\text { Soluble intercellular cell adhesion } \\
\text { molecule-1 (sICAM-1), soluble } \\
\text { platelet selectin (sP-selectin), CD40 } \\
\text { ligand (sCD40 L), platelet factor- } 4 \\
\text { (PF-4) and homocysteine; combined } \\
\text { high-sensitivity C-reactive protein } \\
\text { (hsCRP), interleukin- } 1 \alpha \text { and -6 } \\
\text { (IL- } 1 \alpha \text { and IL-6, respectively) and } \\
\text { tumor necrosis factor- } \alpha \text { (TNF- } \alpha \text { ). }\end{array}$ & $\begin{array}{c}\text { Correlation with radiological } \\
\text { status }\end{array}$ & $\begin{array}{l}\text { Lacunes are associated with different } \\
\text { inflammatory markers. }\end{array}$ \\
\hline $\begin{array}{c}\text { Yang TT et al., } 2018 \\
\text { [51] }\end{array}$ & $\begin{array}{c}101 \mathrm{MCI}, 107 \mathrm{AD}, 30 \text { Parkinson's } \\
\text { disease with dementia (PDD), } 20 \mathrm{VaD} \\
\text { patients }\end{array}$ & $\begin{array}{l}\text { Serum levels of exosomal miR-135a, } \\
-193 \mathrm{~b} \text {, and }-384\end{array}$ & Differential diagnosis & $\begin{array}{l}\text { Both serum exosome miR-135a and } \\
\text { miR-384 were up-regulated while } \\
\text { miR-193b was down-regulated in AD } \\
\text { patients compared with normal controls } \\
\text { and non-AD dementias. }\end{array}$ \\
\hline $\begin{array}{c}\text { Staszewski et al., } 2019 \\
\text { [52] }\end{array}$ & $\begin{array}{c}130 \text { patients with marked MRI features } \\
\text { of SVD and recent lacunar stroke }(n= \\
52, \mathrm{LS}) \text {, vascular Parkinsonism }(n= \\
28, \mathrm{VaP}) \text { or dementia }(n=50, \mathrm{VaD}) .\end{array}$ & $\begin{array}{c}\text { IL-1 } \alpha \text {, IL-6, hs-CRP, sICAM-1, } \\
\text { sP-selectin, TNF- } \alpha \text {, homocysteine, } \\
\text { fibrinogen, D-dimer, serum total } \\
\text { cholesterol (TC), high density } \\
\text { lipoprotein cholesterol (HDL-C), } \\
\text { low-density lipoprotein cholesterol } \\
\text { (LDL-C), triglycerides (TG), eGFR, } \\
\text { serum FG, HbA1c, albumin and } \\
\text { uric acid (UA). }\end{array}$ & $\begin{array}{l}\text { Risk Factor (mean follow-up } \\
\text { time: } 22.3 \pm 4.3 \text { months) }\end{array}$ & $\begin{array}{l}\text { IL- } 1 \alpha, \text { IL- } 6 \text {, homocysteine, d-dimer were } \\
\text { significantly associated with the event of } \\
\text { death or stroke, even after adjusting for } \\
\text { age, sex and SVD radiological markers. }\end{array}$ \\
\hline
\end{tabular}


Table 2. CSF biomarkers and VCID.

\begin{tabular}{|c|c|c|c|c|}
\hline $\begin{array}{l}\text { Author, Year } \\
\text { [Reference] }\end{array}$ & Population & Biomakers & Outcome & Results \\
\hline $\begin{array}{c}\text { Busse S et al., } 2014 \\
\text { [53] }\end{array}$ & $\begin{array}{l}\text { serum and CSF of } 24 \text { patients with } \mathrm{AD}, 20 \\
\text { patients with subcortical ischemic vascular } \\
\text { dementia (SIVD) and } 274 \text { healthy volunteers }\end{array}$ & $\begin{array}{c}\text { N-methyl-D-aspartate } \\
\text { glutamate receptors (NMDA-R) } \\
\text { autoantibodies directed against } \\
\text { the NR1a subunit (NR1a } \\
\text { NMDA-R autoantibodies) }\end{array}$ & $\begin{array}{l}\text { Differential } \\
\text { diagnosis }\end{array}$ & $\begin{array}{c}\text { The overall seroprevalence was not } \\
\text { statistically different between dementia } \\
\text { patients and matched controls. } \\
\text { CSF samples were negative for NMDA-R } \\
\text { autoantibodies. }\end{array}$ \\
\hline $\begin{array}{l}\text { Herbert MK et al., } 2014 \\
\text { [54] }\end{array}$ & $39 \mathrm{DLB}, 110 \mathrm{AD}, 24 \mathrm{VaD}$ and 28 FTD patients. & $\begin{array}{c}\text { CSF concentration of } \\
\text { amyloid-b42 (Ab42), total tau } \\
\text { protein (t-tau), and } \\
\text { phosphorylated tau protein } \\
\text { (p-tau) and 3-methoxy-4- } \\
\text { hydroxyphenylethyleneglycol } \\
\text { (MHPG) }\end{array}$ & $\begin{array}{l}\text { Differential } \\
\text { diagnosis }\end{array}$ & $\begin{array}{l}\text { The used combination of Ab42, } \mathrm{t} \text {-tau, and } \\
\text { p-tau could not discriminate among DLB, } \\
\text { VaD and FTD. } \\
\text { The addition of MHPG to Ab42, } \mathrm{t} \text {-tau, and } \\
\text { p-tau improves the discrimination of DLB } \\
\text { from AD, but could not distinguish DLB } \\
\text { from other forms of dementia. }\end{array}$ \\
\hline $\begin{array}{l}\text { Hermann P et al., } 2014 \\
\text { [55] }\end{array}$ & $\begin{array}{l}32 \text { patients with VaD with Cerebral Small } \\
\text { Vessels Disease (CSVD); } 27 \text { patients with AD; } \\
27 \text { patients with AD + CSVD on MRI. }\end{array}$ & CSF albumin ratio & $\begin{array}{l}\text { Differential } \\
\text { diagnosis }\end{array}$ & $\begin{array}{l}\mathrm{VaD}+\mathrm{CSVD} \text { and } \mathrm{AD}+\mathrm{CSVD} \text { had a higher } \\
\text { albumin ratio, as an expression of } \mathrm{BBB} \\
\text { disruption. }\end{array}$ \\
\hline $\begin{array}{c}\text { Skillbäck T et al., } 2014 \\
\text { [56] }\end{array}$ & $\begin{array}{l}107 \text { healthy controls, } 223 \text { early onset AD, } 1194 \\
\text { late onset AD, } 437 \text { subjects with dementia with } \\
\text { no other specification,146 FTD, } 114 \text { DLB, } 517 \\
\text { MD, } 45 \text { PDD, } 465 \mathrm{VaD}, 108 \text { Other) }\end{array}$ & $\begin{array}{l}\text { CSF neurofilament light (NFL) } \\
\text { levels }\end{array}$ & $\begin{array}{l}\text { Differential } \\
\text { diagnosis }\end{array}$ & $\begin{array}{c}\text { CSF NFL differed among clinical diagnoses, } \\
\text { with the highest levels seen in } \\
\text { frontotemporal dementia, VaD, and mixed } \\
\text { dementia }\end{array}$ \\
\hline $\begin{array}{c}\text { Ewers M et al., } 2015 \\
\text { [57] }\end{array}$ & $\begin{array}{c}55 \text { healthy controls (HC) subjects, } 167 \text { patients } \\
\text { with AD dementia, } 172 \text { subjects with MCI, } 22 \\
\text { subjects with subjective memory impairment } \\
\text { (SMI), } 69 \text { patients with VaD, } 26 \text { patients with } \\
\text { Lewy body dementia (LBD), } 39 \text { patients with } \\
\text { FTD, } 39 \text { patients with depression, and } 86 \\
\text { patients with other neurological disorders } \\
\text { (OND). }\end{array}$ & $\begin{array}{l}\text { CSF concentrations of Ab1-42, } \\
\text { p-tau181, and total tau. }\end{array}$ & $\begin{array}{l}\text { Differential } \\
\text { diagnosis }\end{array}$ & $\begin{array}{l}\text { CSF A } \beta 1-42 \text { showed the best diagnostic } \\
\text { accuracy among the CSF biomarkers. CSF } \\
\text { A } \beta 1-42 \text { discriminates AD dementia from } \\
\text { FTD but shows significant overlap with } \\
\text { other non-AD forms of dementia, possibly } \\
\text { reflecting the underlying mixed } \\
\text { pathologies. }\end{array}$ \\
\hline $\begin{array}{l}\text { Liguori C et al., } 2015 \\
\qquad[58]\end{array}$ & $\begin{array}{l}\text { Patients with } \mathrm{AD}(N=145) \text {, healthy controls } \\
(N=80) \text { and patients with } \operatorname{VaD}(N=44)\end{array}$ & $\begin{array}{l}\text { CSF lactate concentrations, AD } \\
\text { biomarker levels ( } \tau \text {-proteins } \\
\text { and } \beta \text {-amyloid) }\end{array}$ & $\begin{array}{l}\text { Differential } \\
\text { diagnosis }\end{array}$ & $\begin{array}{l}\text { AD patients showed a significant increase } \\
\text { of CSF lactate concentration compared to } \\
\text { controls and patients with VaD. }\end{array}$ \\
\hline
\end{tabular}


Table 2. Cont.

\begin{tabular}{|c|c|c|c|c|}
\hline $\begin{array}{c}\text { Author, Year } \\
\text { [Reference] }\end{array}$ & Population & Biomakers & Outcome & Results \\
\hline $\begin{array}{c}\text { Rosenberg GA et al., } \\
2015 \\
{[59]}\end{array}$ & $\begin{array}{l}62 \text { patients with Vascular Cognitive } \\
\text { Impairment (VCI) }\end{array}$ & $\begin{array}{l}\text { CSF measurements of albumin } \\
\text { ratio, matrix metalloproteinases } \\
\text { (MMPs), amyloid- } \beta 1-42 \text { and } \\
\text { phosphorylated- } \tau 181 . \\
\text { Patients were followed for an } \\
\text { average of } 2 \text { years. }\end{array}$ & Predictor & $\begin{array}{c}\text { Inflammatory biomarkers of increased BBB } \\
\text { permeability, elevated albumin index and } \\
\text { reduced MMP-2 index, predicted the } \\
\text { diagnosis of the Binswanger disease (BD) } \\
\text { type of subcortical ischaemic vascular } \\
\text { disease. }\end{array}$ \\
\hline $\begin{array}{c}\text { Skillbäck T et al., } 2015 \\
\text { [60] }\end{array}$ & $\begin{array}{l}383 \text { Early onset } \mathrm{AD} \text { and } 221 \text { late onset } \mathrm{AD} \\
\text { patients, } 759 \text { vascular dementia, } 982 \text { mixed } \\
\text { dementia, } 232 \text { frontotemporal dementia, } 150 \\
\text { Parkinson's disease dementia and } 79 \text { dementia } \\
\text { with Lewy bodies }(N=79) \text {. }\end{array}$ & $\begin{array}{l}\text { cerebrospinal fluid } \\
\text { amyloid- } \beta 1-42 \text {, total tau and } \\
\text { phosphorylated tau. }\end{array}$ & $\begin{array}{c}\text { Differential } \\
\text { diagnosis }\end{array}$ & $\begin{array}{c}\text { In Parkinson's disease dementia and } \\
\text { vascular dementia low CSF amyloid- } \beta 1-42 \\
\text { was associated with low Mini-Mental State } \\
\text { Examination score. }\end{array}$ \\
\hline $\begin{array}{c}\text { Struyfs H et al., } 2015 \\
{[61]}\end{array}$ & $\begin{array}{l}\text { Patients with AD }(n=50), \text { MCI due to AD }(n= \\
50) \text { and patients with non-AD dementias }(n= \\
\text { 50). The non-AD group consisted of } 17 \text { patients } \\
\text { with FTD, } 17 \text { DLB patients, and } 16 \text { patients } \\
\text { with vascular dementia (VaD). The Control } \\
\text { group was composed of } 35 \text { subjects. }\end{array}$ & $\begin{array}{c}\text { CSF levels of } A \beta \text { isoforms, } \\
\mathrm{A} \beta(1-37), \mathrm{A} \beta(1-38) \text {, and } \\
\mathrm{A} \beta(1-40) \text {, as compared to the } \\
\text { AD CSF biomarkers } A \beta(1-42), \\
\text { T-tau, and P-tau(181P). }\end{array}$ & $\begin{array}{c}\text { Differential } \\
\text { diagnosis }\end{array}$ & $\begin{array}{l}\text { Best biomarkers to distinguish } A D \text { and } V a D \\
\text { were } A \beta 1-42 / T \text {-tau and } A \beta 1-42 / P \text {-tau181P }\end{array}$ \\
\hline $\begin{array}{c}\text { Skillbäck T et al., } 2017 \\
\text { [62] }\end{array}$ & $\begin{array}{c}\text { Patients diagnosed with Alzheimer's disease } \\
\text { (AD, early onset [EAD, } n=130] \text {, late onset AD } \\
\text { [LAD, } n=666] \text { ), vascular dementia (VaD, } n= \\
\text { 255), mixed AD and VaD (MD, } n=362) \text {, Lewy } \\
\text { body dementia (DLB, } n=50) \text {, frontotemporal } \\
\text { dementia (FTD, } n=56), \text { Parkinson's disease } \\
\text { dementia (PDD, } n=23) \text {, other dementias } \\
\text { (other, } n=48), \text { and dementia not otherwise } \\
\text { specified (NOS, } n=271) \text {, two healthy control } \\
\text { groups (n=292, } n=20 \text { ). }\end{array}$ & CSF/serum albumin ratio & $\begin{array}{l}\text { Differential } \\
\text { diagnnosis }\end{array}$ & $\begin{array}{c}\text { Patients with DLB, LAD, VaD, MD, other, } \\
\text { and NOS groups had higher CSF/serum } \\
\text { albumin ratio than controls. } \\
\text { CSF/serum albumin ratio correlated with } \\
\text { CSF neurofilament light in LAD, MIX, VaD, } \\
\text { and other groups but not with AD } \\
\text { biomarkers. }\end{array}$ \\
\hline
\end{tabular}


Table 2. Cont.

\begin{tabular}{|c|c|c|c|c|}
\hline $\begin{array}{c}\text { Author, Year } \\
\text { [Reference] }\end{array}$ & Population & Biomakers & Outcome & Results \\
\hline $\begin{array}{c}\text { Kiđemet-Piskač S et al., } \\
2018 \\
{[63]}\end{array}$ & $\begin{array}{l}152 \text { patients with } \mathrm{AD}, 28 \mathrm{VaD} \text {, and } 18 \text { healthy } \\
\text { controls }(\mathrm{HC}) \text {. }\end{array}$ & $\begin{array}{l}\text { CSF levels of total tau protein } \\
\text { (t-tau), tau protein } \\
\text { phosphorylated at threonine } 231 \\
\quad(\mathrm{p} \text {-tau231). }\end{array}$ & $\begin{array}{l}\text { Differential } \\
\text { diagnosis }\end{array}$ & $\begin{array}{l}\text { Total tau levels were significantly elevated } \\
\text { in subjects with AD compared to } \mathrm{HC} \text {, as } \\
\text { well as in VaD subjects compared to HC. } \\
\text { p-tau }{ }_{231} \text { levels were significantly higher in } \\
\text { patients with AD vs. HC as well in patients } \\
\text { with VaD vs. HC. } \\
\text { p-tau231 levels did not distinguish AD } \\
\text { from VaD patients. }\end{array}$ \\
\hline $\begin{array}{c}\text { Barry Erhardt E et al., } \\
2018 \\
{[64]}\end{array}$ & 62 possible VCID patients & $\begin{array}{l}\text { Matrix metalloproteinases-2 } \\
\text { (MMP-2) and MMP-9 in the CSF } \\
\text { and plasma and MMP-2 and } \\
\text { MMP-9 indexes were calculated. } \\
\text { Amyloid b1-42 (Ab42) and } \\
\text { phosphoTau181 (PTau) in the } \\
\text { CSF. }\end{array}$ & Diagnostic accuracy & $\begin{array}{c}\text { MMP-2 was accurate in predicting VCID } \\
\text { diagnosis }\end{array}$ \\
\hline $\begin{array}{c}\text { Chakraborty A et al., } \\
2018 \\
{[65]}\end{array}$ & $\begin{array}{l}\text { age-matched groups of controls with subjective } \\
\text { cognitive decline }(n=21) \text {, AD without the } \\
\text { presence of microbleeds } \\
(\mathrm{MB})(n=25), \mathrm{AD} \text { with } \mathrm{MB}(n=25) \text {, and VaD } \\
(n=21) \text { patients. }\end{array}$ & VEGF levels in CSF & $\begin{array}{l}\text { Differential } \\
\text { diagnosis }\end{array}$ & $\begin{array}{l}\text { No significant differences were detected } \\
\text { between groups }\end{array}$ \\
\hline
\end{tabular}


Neuroimaging and neuropathology studies have established that clinically silent CVD is sufficient to cause relevant cognitive impairment in the absence of stroke [5]. SVD, characterised by arteriolosclerosis and lacunar infarcts, causes cortical and subcortical microinfarcts, which appear to be the most robust pathological substrates of VCID [14]. Indeed, diffuse damage to the white matter (ischemic leukoencephalopathy) is the most common pathology in SVD [66]. It is usually most severe in frontal and occipital regions and the area involved shows loss of both myelin and axons as well as chronic inflammatory infiltrates [67]. The mechanism underlying white matter lesions (WMLs), which lead to demyelination and gliosis, involves a multifactorial process, including BBB disruption, hypoxia and hypoperfusion, oxidative stress, neuroinflammation and alteration on NVU coupling [15]. In addition to lacunar infarcts and WMLs, brain atrophy and visible Enlarged Perivascular spaces (EPVS) are other common radiologic features of SVD. Their relationship to vascular cognitive impairment, however, is not well established [68]. Recent pathological data show that SVD may be linked to the dysfunction of the glymphatic pathway that might be involved in the clearance of mis-aggregated proteins. On this basis, EPVS, identifiable on brain MRI, have been described as a potential biomarker of neurovascular dysfunction and of impaired clearance of proteins [68,69]. Finally, hemorrhagic manifestations such as cerebral microbleeds or superficial siderosis are other possible causes of cognitive impairment in SVD [14].

As we mentioned above, SVD is one of the major causes of VCID and it is usually a sporadic disease caused by age and hypertension [70], however there are also monogenic forms of SVD. The most common form is the Cerebral Autosomal Dominant Arteriopathy with Subcortical Infarcts and Leukoencephalopathy (CADASIL), caused by mutations in the NOTCH3 gene [71]. Other rare forms include the Cerebral Autosomal Recessive Arteriopathy with Subcortical Infarcts and Leukoencephalopathy (CARASIL), an arteriopathy caused by mutations in the HTRA1 gene [72] and the Cathepsin A-Related Arteriopathy with Strokes and Leukoencephalopathy (CARASAL), an autosomal dominant disease caused by heterozygous mutation in the CTSA gene encoding cathepsin A [73]. Moreover, other adult-onset genetic leukoencephalopathies have recently been described, such as Hereditary Diffuse. Leukoencephalopathy with Spheroids (HDLS), caused by mutations in the CSF1R (Colony Stimulating Factor-1 Receptor) gene and responsible of approximately $10 \%$ of cases of adult-onset leukodystrophy [74]. It is important to recognize these forms of SVD to make an accurate diagnosis and a correct family counseling.

\section{Endothelial Dysfunction}

The endothelium is a monolayer of endothelial cells that separates the tissues from the circulating blood. It is involved in several vascular processes and functions. The term endothelial dysfunction indicates a condition in which the endothelium is damaged and can develop proinflammatory, provasoconstriction and procoagulation states [75].

As we mentioned above, most of VCID is caused by cerebral hypoperfusion [76]. Cerebrovascular endothelial cells are usually the foremost in bearing the attack of hypoperfusion. The subsequent endothelial dysfunction could lead to many processes such as an increase in BBB permeability, an exposure of neural cells to harmful substances, a rise of inflammatory environment and a dysfunction of neurovascular coupling. The result is an activation of glial cells in the CNS, which lead to WMLs and neuron damage [77]. An Australian study showed that reduced endothelial integrity was associated with increasing WMHs severity [78]. Moreover, a significant decrease in endothelial function and in BBB integrity are present in areas of WMHs compared with normal white matter [78]. This hypothesis is supported by neuropathological studies, showing that WMLs are characterized by the expression of hypoxia-related molecules and endothelial markers [79]. Cerebral hypoperfusion up-regulates the expression of adhesion molecules such as intercellular adhesion molecule-1 (ICAM-1) and vascular cell adhesion molecule-1 (VCAM-1), markers of endothelial cell activation [80]. Finally, endothelial dysfunction promotes oxidative stress from unbalanced free radical formation, which leads 
to peroxynitrite formation, lipid peroxidation, protein modification, matrix metalloproteinases (MMPs) activation, and DNA damage [13].

\section{Blood-Brain Barrier and Choroid Lexus Breakdown}

The BBB isolates the central nervous system (CNS) from the systemic circulation and it is essential in maintaining the optimal microenvironment in the CNS. It is maintained by the interplay between endothelial cells, pericytes, and astrocytes [81].

The choroid plexus is an epithelial monolayer that forms the blood-cerebrospinal fluid barrier. The main function of choroid plexus is to produce CSF, but it also acts as a neuro-immune interface by integrating signals from the brain with signals coming from the circulation. The CSF/serum albumin ratio is the gold-standard measure of $\mathrm{BBB}$ and choroid plexus integrity, with increases in this ratio indicating increased permeability. Disruption of BBB and choroid plexus functions, followed by a rise of neuroinflammatory molecules coming from blood circulation, may promote brain damage [67]. These events may be involved in disease progression in both VCID and AD [82].

Nowadays, it is well known that BBB permeability is altered in patients with cerebral microvascular disease. A recent meta-analysis [83] revealed that BBB permeability was increased further in patients with either VaD or AD compared with age-matched healthy controls. Moreover, the permeability was higher in VaD compared with AD.

\section{The Role of Inflammation}

Inflammation has been recognized to have a crucial role in the pathogenic mechanisms of cerebrovascular and neurodegenerative diseases [84]. It is not yet known whether inflammation is a primary driver of VCID and whether this neuroinflammation is triggered by intrinsic or systemic processes [80]. Certainly, clinical observations suggest that an interplay between CNS and peripheral inflammation exists [85]. Microvascular inflammation, as outlined previously, is a common feature of hypoperfusion models with markers of chronic inflammation and endothelial activation, leading to increased BBB permeability and to infiltration of inflammatory factors like interleukins (ILs), MMPs, Tumor necrosis factor (TNF $\alpha$ ), toll-like receptor 4 (TLR4), C-reactive protein (CRP). Upon entry into the brain, these inflammatory factors can exacerbate white matter damage (demyelination, axonal loss, oligodendrocyte degeneration), cause neurodegeneration and cell death as well as enhance neuroglial inflammation [86] (Figure 1). Indeed, clinical and experimental studies have shown that white matter damage is not merely a consequence of chronic oxygen deprivation but is induced and sustained by pro-inflammatory environments [85]. In the hippocampus, as a result of the inflammatory cascade, it was observed an impaired neurogenesis, a dysregulation of progenitor cell proliferation and an alteration of synaptic plasticity and dendritic spine density [86].

Several other processes are promoted by inflammation and might contribute to the pathogenesis of VCID such as atherogenesis and platelet aggregation. Cytokines, leukocyte adhesion molecules, chemokines, growth factors, and lipids mediate these processes [13]. 


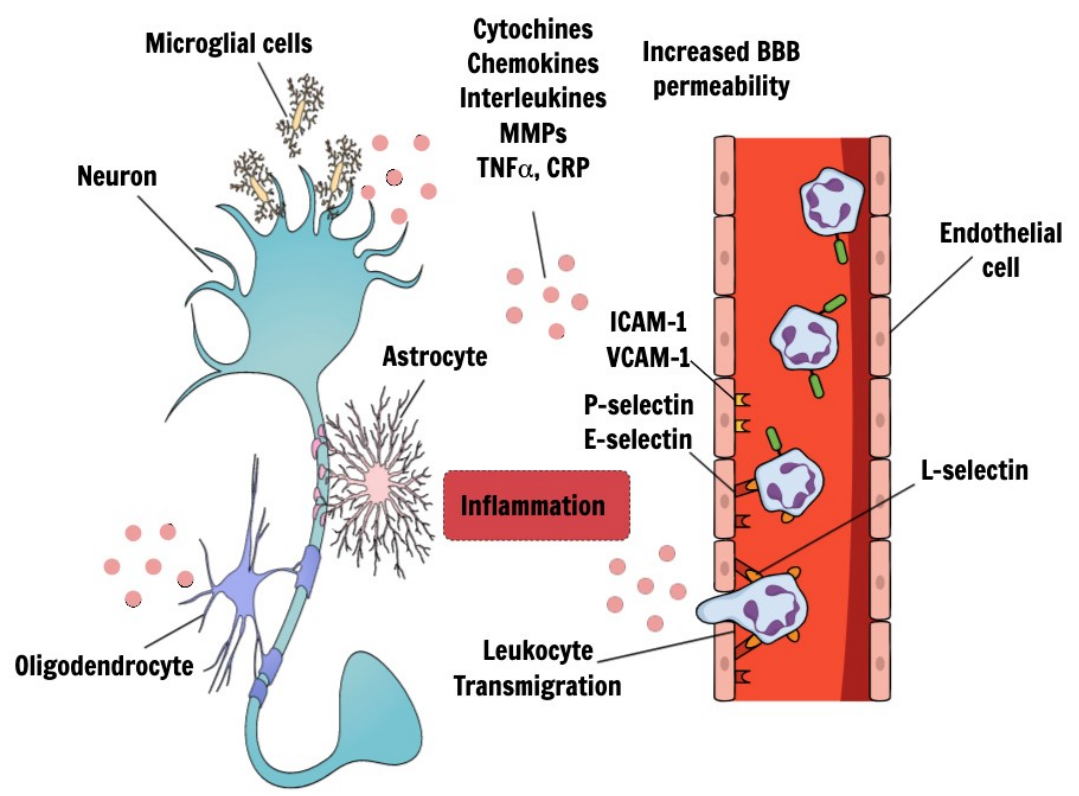

Figure 1. Endothelial dysfunction and cerebral inflammatory response in VCID. Endothelial dysfunction, observed in VCID, could lead to an increase in BBB permeability, a subsequent exposure of neural cells to harmful substances and a rise of inflammatory environment. The result is an activation of glial cells in the CNS, which finally lead to neuron damage. During inflammatory response, leukocytes are recruited from the circulation into the extravascular space. The activation of endothelial cells lead to the expression of adhesion molecules on their surfaces, allowing the binding to reciprocal molecules on the surfaces of circulating leukocytes. Indeed, activated endothelial cells up-regulate the expression of adhesion molecules such as intercellular adhesion molecule-1 (ICAM-1), vascular cell adhesion molecule-1 (VCAM-1) and selectins (P-selectin, E-selectin). L-selectin is mainly expressed on leukocytes. The increased BBB permeability lead to infiltration of inflammatory factors like interleukins, chemokines, cytochines, MMPs, TNF $\alpha$, TLR4, CRP. Upon entry into the brain, these inflammatory factors can exacerbate white matter damage (demyelination, axonal loss, oligodendrocyte degeneration), cause neurodegeneration and cell death as well as enhance neuroglial inflammation. (VCID: vascular cognitive impairment and dementia; BBB: blood-brain barrier; CNS: central nervous system; MMPs: $\mathrm{TNF} \alpha$ : Tumor necrosis factor; CRP: C-reactive protein).

\section{Biomarkers}

Identifying a diagnostic biomarker in VCID would be of paramount interest in order to contribute to a better understanding of the mechanisms underlying this affection. The goal of diagnostic biomarkers development in VCID is to identify patients at early stage when treatment may be effective in blocking the progressive damage to the white matter. Nevertheless, it may be also desirable to find biomarkers that allow the clinician to follow the natural history of the disease, in terms of severity and prognosis.

An accurate biomarker-such as a protein, nucleic acid, or metabolite-should represent a quantification of a definite biological state. Due to its heterogeneity, VCID may not have a uniform presentation and a linear progress over time, so biomarkers may differ depending on aetiology, clinical phase and histopathologic involvement. Given these premises, it is likely that a single biomarker might not be adequate to identify underlying complexities that are at the basis of cellular changes linked to VCID. Furthermore, it has to be reminded that in clinical practice a circulating biomarker has to be easily assessed with low cost, and patient-friendly procedure. In this light, several families of candidate biomarkers have been studied in relation to VCID and multiple approaches have been developed to identify biomarkers [87]. To facilitate a thorough comprehension of their significance in terms of aetiology, they could be categorized in four subgroups:

(1) Biomarkers of Inflammatory Response; 
(2) Biomarkers of Central Nervous System Tissue Injuries;

(3) Biomarkers of Coagulation and Thrombosis;

(4) Circulatory miRNAs.

\subsection{Biomarkers of Inflammatory Response}

Strong evidences suggest that chronic inflammation is involved in the pathogenesis of several conditions such as stroke, dementia, cardiovascular disease and atherosclerosis [88]. However, the data regarding the association between inflammatory biomarkers and VCID are still inconclusive.

Considering SVD, several studies found an association between inflammatory biomarkers and the progression of the disease. In this context, the vascular damage observed in the brain may be promoted and sustained by cytokines, acute-phase proteins, endothelial cell adhesive molecules, and other immune-related proteins (Figure 1). However, due to the lack of consensus on analytic methods, the results are often inconsistent [89]. Traditionally, the most commonly studied biomarkers in this field are interleukin-6 (IL-6) and CRP [90].

IL-6 is secreted as a pro-inflammatory cytokine by tunica muscularis cells of the blood vessels. In MRI studies, a positive correlation between IL-6 and WMHs has been detected [91]. As a response to IL-6, the liver synthesizes the CRP, which increases BBB permeability. On these bases, it has been suggested that CRP may mirror the inflammatory process in the brain and described that higher CRP levels are associated with increased risk of dementia [92]. It has also been reported an association between typical MRI findings in VCID such as WMHs, ischemic lacunes and EPVS and higher CRP levels [92-94]. Furthermore, a rapid decline in CRP levels predicts a healthier white matter microstructure [95]. However, the association between CRP and WMHs was reconsidered by the adjustment of cardiovascular factors [96]. In contrast to these results, other studies did not find any associations between CRP or other cytokines and the severity of WMHs volume in VCID [89].

Inflammatory biomarkers other than CRP or IL-6 may be relevant for WMHs in VCID. Indeed, higher levels of several endothelial dysfunction markers such as soluble E-selectin, soluble intercellular adhesion molecule 1, soluble P-selectin, and soluble vascular cell adhesion molecule-1 have been related with WMHs in cross-sectional studies [97,98]. Moreover, Gu et al. [89] found that alpha 1-antichymotrypsin (ACT), which plays a role in cell adhesion and endothelial dysfunction, was associated with both WMHs severity and WMHs progression [89].

MMPs are other biomarkers of inflammation. They are a family of 26 extracellular matrix-degrading enzymes that attack the basal lamina and tight junctions of the cerebral endothelial cells, causing BBB dysfunction. They have been identified in pathological tissues from animal models of VCID and in human tissues [59,99]. Some MMPs, such as MMP-2, are normally present in the CSF. Others (mainly MMP-3 and MMP-9) are present at very low levels in the CSF in the absence of an inflammatory state [100]. Some studies have demonstrated that the evaluation of MMPs in CSF have high validity in discriminating VCID from cognitive impairment of primarily neurodegenerative etiology [12,101,102]. Indeed, elevated CSF levels of MMP-9 are present in patients with VCID and mixed AD/VCID but not in AD [12]. MMPs were explored also in plasma levels. A work by Duits et al. [19] showed how they can help differentiate VaD from AD. Given these evidences, MMPs could be promising biomarkers in the classification of the different types of dementia.

\subsection{Biomarkers of Central Nervous System Tissue Injuries}

Numerous biomarkers have been associated with CNS tissue injury, including neuron specific enolase (NSE), $N$-methyl-D-aspartate receptor (NMDAR) antibodies, protein S-100B, and myelin basic protein (MBP).

NMDAR are both ligand-gated and voltage dependent and are involved in long-term potentiation in learning processes of the brain [103]. Autoantibodies directed to some NMDAR subtypes have been mainly studied in acute cerebrovascular events, but also in post-stroke cognitive impairment. Busse and colleagues [53] examined the prevalence of NR1a NMDAR autoantibodies in both serum and CSF 
of subjects affected by AD, SVD and healthy subjects. The results of the study showed the presence of NMDAR IgM, IgG, and/or IgA autoantibody titers in serum of patients with SVD. However, the results were not conclusive, because the seroprevalence of NMDAR-directed autoantibodies was age related [53].

MBP is a hydrophilic protein found in myelin sheaths, whose serum levels have been found in several neurological diseases. At this regard myelin loss has been found in different types of dementia. Literature data suggest that myelin loss, secondary to hypoxic-ischemic damage, may evolve in parallel with shrunken oligodendrocytes in $\mathrm{VaD}[104,105]$.

$\mathrm{S} 110 \mathrm{~B}$ is an astroglial protein that has been studied as a serum marker for cerebral injury and disruption of the BBB. It has been studied as an independent predictor and diagnostic marker for stroke, VCID, and AD. However, no significant difference in S100B CSF levels was found between AD and VCID patients [106].

Neurofilament light chain $(\mathrm{NfL})$ is an emerging blood and CSF biomarker for neuroaxonal damage. Its role has been explored in multiple neurological diseases affecting the elderly population, such as motor neuron disease, AD and frontotemporal dementia [56]. A recent study found, in both sporadic SVD and CADASIL patients, an association between serum NfL levels and impaired processing speed performance. Furthermore, CADASIL patients showed a strong and independent association between serum NfL levels and measures of focal neurological deficits and disability [107].

The results of another study suggest that in CADASIL the serum NfL levels correlate with disease severity, disease progression and 17-year survival [108]. Given these evidences, in CADASIL patients serum NfL could be a promising biomarker to monitor disease course and a possible target for new therapies.

Finally, evidences on CSF levels of $A \beta$ and Tau proteins reveal how they may be of help in differentiating VCID from $A D$, even if there is a certain degree of overlap between $A D$ and non-AD dementias, possibly reflecting underlying mixed pathologies [54,57,59-61].

\subsection{Biomarkers of Coagulation and Thrombosis}

It is well known that coagulation and fibrinolytic pathways are involved in cerebrovascular diseases. However, little is known about the role of haemostatic biomarkers in VCID with most of the studies yielding discordant evidences [13].

Biomarkers identifying the coagulation cascade have been linked to stroke, VaD and AD: such biomarkers include, among the others, fibrinogen and lipoprotein-associated phospholipase A2 (Lp PLA2).

Fibrinogen is a soluble plasma glycoprotein involved in the coagulation cascade. Some studies suggest that high fibrinogen levels correlate with increased risk of dementia, both $\mathrm{AD}$ and $\operatorname{VaD}[109,110]$. This is supported by a study on MCI patients, which observed that hyperfibrinogenemia was associated with an increased risk for developing $\mathrm{VaD}$ [111].

Lp PLA2 is an enzyme expressed primarily by leukocytes that influence the degradation of platelet-activating factors to inactive factors. It plays a crucial rule in the metabolism of low-density lipoprotein to proinflammatory proteins. Lp PLA2 is expressed in the necrotic core of atherosclerotic plaques, thus resulting a valid biomarker of atherosclerosis and plaque inflammation. However, an increased risk of dementia has also been associated with eleveted Lp PLA2 activity [112].

It is known that prothrombotic status is associated with cardiovascular risk. Moreover, tissue factor and thrombomodulin, markers of endothelial activation and damage, have been associated with the extent of leukoaraiosis in SVD [113].

Several studies found an association of high plasma levels of von Willebrand factor, a glycoprotein expressed by endothelial cells after tissue damage, with the number of lacunes, periventricular WMHs, and WMHs burden $[114,115]$. Moreover, in CADASIL patients, von Willebrand factor was significantly higher than in controls [116]. 
Different authors described an association between high homocysteine levels, depending on metabolism of dietary methionine, and WMHs or silent lacunar infarcts [75]. In a large cohort of CADASIL patient, hyperhomocysteinemia was associated with an increased risk of migraine [117].

Endothelial progenitor cells (EPCs) and circulating progenitor cells (CPCs) are bone marrow-derived cells involved in the maintenance of the structure and homeostasis of the endothelium [118]. Lower levels of EPCs are a strong predictor of cardiovascular events and some studies reported lower levels of EPCs in CADASIL and sporadic SVD patients [118]. These results were confirmed in a larger cohort of CADASIL patients [116]. About the CPCs levels in CADASIL patients, they were significantly correlated with a more severe decrease of both cognitive and motor performances [118] as well as with neuroimaging findings [116].

\subsection{Circulating microRNA}

miRNAs are small, noncoding, single-stranded RNA molecules that negatively regulate gene expression via translational inhibition or mRNA degradation. Their biological importance within organisms at all levels of the evolutionary scale has been demonstrated [119]. miRNAs genes are excised from exons and introns or other intergenic regions of the genome [120].

An alteration of miRNAs expression has been observed in several human diseases and it may play a role in CVD, improving the gene-regulatory processes [121]. For these reasons, research on peripheral biomarkers in various human diseases is considering miRNAs [122]. Circulating miRNAs have been detected in plasma, serum, whole blood, urine, saliva, sweat, breath, and CSF, and, because of their small molecular size, they cross biological barriers (e.g., blood/brain, blood/placenta) [123]. Therefore, they have the potential to serve as disease biomarkers, being reported to be reproducible and stable among people [124]. Increasing number of miRNAs have been proven to be critical for the pathogenesis of neurological diseases [125]. Many studies have shown that, after CNS injury, altered miRNAs may activate processes that stimulate neuronal death through inflammation, apoptosis, and oxidative stress [126].

In a recent work by Ragusa et al. [127], miRNA such as miR-10b*, miR29a-3p and miR-130b-3p were downregulated in both $\mathrm{VaD}$ and $\mathrm{AD}$ patients, but the levels of miR-130b-3p were lower in $\mathrm{AD}$ than in VaD patients. Furthermore Dong et al. [18] found that a panel of other three miRNA (miR-31, miR-93 and miR-146a) significantly upregulated in VaD patients, could be used to discriminate AD from VaD.

With the purpose of differentiating VaD from $\mathrm{AD}$, a small exploratory investigation by Sørensen et al. [128] focused on the analysis of miRNA in CSF of patients affected by AD and by other types of dementia. In this exploratory study, deregulated miRNAs in seem to be associated with target genes related to $\mathrm{AD}$ pathology, suggesting that miRNAs are interesting candidates for AD biomarkers in the future.

Finally, Prabhakar et al. [39] revealed that four miRNAs (miR-409-3p, miR-502-3p, miR-486- 5p and miR-451a) could be used as valuable biomarkers to differentiate SVD patients from healthy controls and might serve as diagnostic biomarkers for this disease. Currently, information regarding miRNAs in VCID studies is limited, but the potential diagnostic value of miRNAs is very promising.

\section{Discussion}

We have reviewed blood and CSF biomarkers that have been related to VCID, with both sporadic and genetic SVD. A summary of the best emerging biomarkers is presented on Tables 1 and 2. Evidences from the literature are heterogeneous and mostly inconclusive. This heterogeneity is mainly explained by the differences of studies design, including different cohorts of patients analyzed and different sample sizes. However, it has to be remarked that VCID is a complex syndrome, possibly resultant from a multifactorial source of pathogenic processes. Therefore, a panel of biomarkers that reflects the different pathophysiological characteristics of the disease, might be needed to understand the contributions of each pathway involved in its pathogenesis. 
From the data collected, the stronger results regard inflammatory biomarkers and molecules involved in endothelial dysfunction and coagulation cascade, although some of them have also been described to be altered in AD. This is the case of serum NfL, that is considered a potential circulating biomarker for both sporadic SVD and CADASIL burden [107]. It is a non-specific biomarker for neuroaxonal damage and higher serum levels have been reported also in AD patients. Commonly, the overlap of these biomarkers in VCID and AD may reflect the presence of concomitant AD and VCID neuropathological alterations.

Regarding endothelial circulating biomarkers including EPCs and CPCs, a possible limitation is that their levels in blood samples depend on the status of systemic endothelium and not only of cerebral endothelium [75].

Currently, data regarding miRNAs in VaD and Post Stroke Dementia (PSD) studies are limited, but the potential diagnostic value of miRNAs is very promising. In spite of the rapidly growing number of publications on diagnostic applications of circulating miRNAs, their use for screening of CNS diseases is in early stages of development. One factor impeding the progress in this field is the difficulty of comparing the data reported by different groups due to the use of different search methods and different techniques for miRNA measurement as well as the lack of data normalization.

Another critical issue of the use of circulating miRNAs as biomarkers for diagnosis and e progression of a disease is that the pathologic processes that support the onset of the disease may change during disease progression. Furthermore, some processes, such as destruction of synapses, are common for different pathologies, including normal brain aging and neurodegenerative diseases, making the concept of differential diagnosis in CNS disease more complicated. The detection of specific miRNAs for brain regions and disease stage, preferably with longitudinal studies, might be useful in following the natural history of the disease and might facilitate the development of screening, predictive.

In this review, we do not include the analysis of biomarkers related to different clinical symptoms or VCID phenotypes. We only reported the results coming from studies on monogenic form of SVD, where the population involved is more homogeneous. Of course, biomarkers alone are often not enough to draw conclusions on a diagnosis, so they use should be considered as a support tool for a more thorough patient assessment, in a "difficult" diagnosis.

\section{Conclusions and Future Perspectives}

VCID is a common neurocognitive disorder still under-recognized in clinical practice and not well studied in research contexts. Evidences about CSF and blood-circulating proteins highlight the involvement of several interrelated mechanisms in the pathogenesis of VCID, including neuroinflammation, haemostasis, lipid metabolism and endothelial dysfunction. Starting from these mechanisms, emerging biomarkers have been studied. Most of analyzed studies show significant cross-sectional associations with neuroimaging or cognitive outcomes, but their utility in clinical setting remains to be established.

An important factor that restrict the applications of these biomarkers is the difficulty of comparing the data reported by different groups, due to the use of different laboratory techniques and methods of measurement. A codification for standardized procedures and methods would be of great value to further investigations.

In conclusion, further studies are needed to better understand the underlying mechanisms of tissue injury in VCID. Moreover, there is the necessity to search for an optimal panel of biomarkers with high sensitivity and specificity through a collaborative international network with harmonized protocols and procedures.

Conflicts of Interest: The authors declare no conflict of interest. 


\section{Abbreviations}

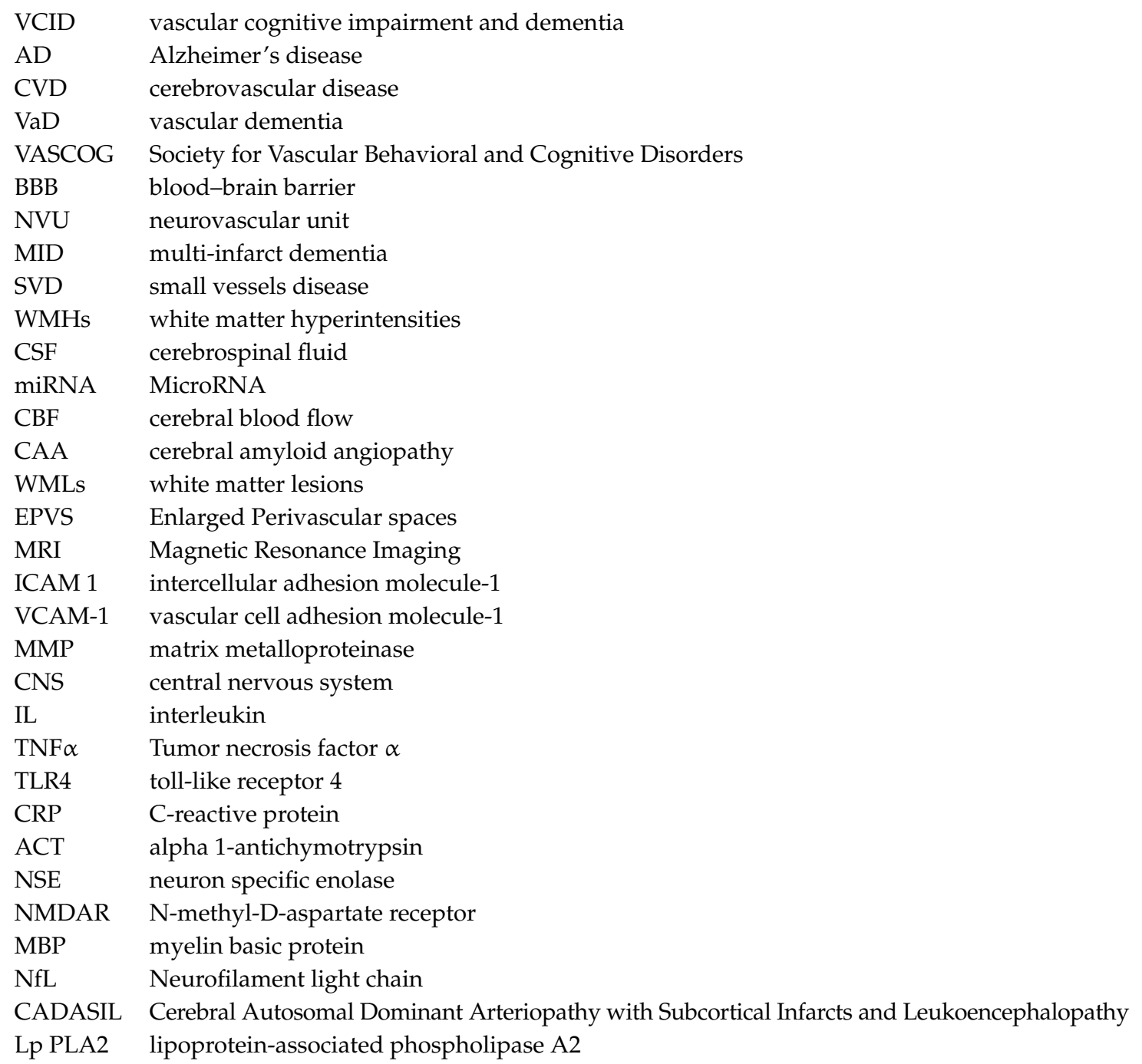

\section{References}

1. Skrobot, O.A.; O’Brien, J.; Black, S.; Chen, C.; DeCarli, C.; Erkinjuntti, T.; Ford, G.A.; Kalaria, R.N.; Pantoni, L.; Pasquier, F.; et al. The Vascular Impairment of Cognition Classification Consensus Study. Alzheimer's Dement. 2016, 13, 624-633. [CrossRef]

2. Murphy, M.P.; Corriveau, R.A.; Wilcock, D.M. Vascular contributions to cognitive impairment and dementia (VCID). Biochim. Biophys. Acta (BBA) - Mol. Basis Dis. 2016, 1862, 857-859. [CrossRef] [PubMed]

3. Lang, B.; Kindy, M.S.; Kozel, F.A.; Schultz, S.K.; Taheri, S. Multi-Parametric Classification of Vascular Cognitive Impairment and Dementia: The Impact of Diverse CerebrovRefascular Injury Biomarkers. J. Alzheimer's Dis. 2018, 62, 39-60. [CrossRef] [PubMed]

4. Paradise, M.B.; Sachdev, P.S. Vascular Cognitive Disorder. Semin. Neurol. 2019, 392, 241-250. [CrossRef]

5. Sachdev, P.; Kalaria, R.; O’Brien, J.; Skoog, I.; Alladi, S.; Black, S.E.; Blacker, D.; Blazer, D.G.; Chen, C.; Chui, H.; et al. Diagnostic criteria for vascular cognitive disorders: A VASCOG statement. Alzheimer Dis. Assoc. Disord. 2014, 28, 206-218. [CrossRef]

6. American Psychiatric Association. Diagnostic and Statistical Manual of Mental Disorders: DSM-V, 5th ed.; American Psychiatric Publishing: Arlington, VA, USA, 2014.

7. Van der Flier, W.M.; Skoog, I.; Schneider, J.A.; Pantoni, L.; Mok, V.; Chen, C.L.H.; Scheltens, P. Vascular cognitive impairment. Nat. Rev. Dis. Primers 2018, 15, 18003. [CrossRef] [PubMed]

8. Gorelick, P.B.; Counts, S.E.; Nyenhuis, D. Vascular cognitive impairment and dementia. Biochim. Biophys. Acta (BBA)—Bioenerg. 2015, 1862, 860-868. [CrossRef] [PubMed] 
9. Hachinski, V.C.; Lassen, N.A.; Marshall, J. Multi-infarct dementia. A cause of mental deterioration in the elderly. Lancet 1974, 2, 207-210. [CrossRef]

10. Rosenberg, G.A. Extracellular matrix inflammation in vascular cognitive impairment and dementia. Clin. Sci. 2017, 131, 425-437. [CrossRef] [PubMed]

11. Hachinski, V.; World Stroke, O. Stroke and Potentially Preventable Dementias Proclamation: Updated World Stroke Day Proclamation. Stroke 2015, 46, 3039-3040. [CrossRef]

12. Rosenberg, G.A.; Bjerke, M.; Wallin, A. Multimodal markers of inflammation in the subcortical ischemic vascular disease type of vascular cognitive impairment. Stroke 2014, 45, 1531-1538. [CrossRef] [PubMed]

13. Vilar-Bergua, A.; Riba-Llena, I.; Nafría, C.; Bustamante, A.; Llombart, V.; Delgado, P.; Montaner, J. Blood and CSF biomarkers in brain subcortical ischemic vascular disease: Involved pathways and clinical applicability. J. Cereb. Blood Flow Metab. 2016, 36, 55-71. [CrossRef] [PubMed]

14. Kalaria, R.N. Neuropathological diagnosis of vascular cognitive impairment and vascular dementia with implications for Alzheimer's disease. Acta Neuropathol. 2016, 131, 659-685. [CrossRef] [PubMed]

15. Yang, T.; Sun, Y.; Lu, Z.; Leak, R.K.; Zhang, F. The impact of cerebrovascular aging on vascular cognitive impairment and dementia. Ageing Res. Rev. 2017, 34, 15-29. [CrossRef] [PubMed]

16. McAleese, K.E.; Alafuzoff, I.; Charidimou, A.; de Reuck, J.; Grinberg, L.T.; Hainsworth, A.H.; Hortobagyi, T.; Ince, P.; Jellinger, K.; Gao, J.; et al. Post-mortem assessment in vascular dementia: Advances and aspirations. BMC Med. 2016, 14, 13. [CrossRef] [PubMed]

17. Murr, J.; Carmichael, P.-H.; Julien, P.; Laurin, D. Plasma oxidized low-density lipoprotein levels and risk of Alzheimer's disease. Neurobiol. Aging 2014, 35, 1833-1838. [CrossRef]

18. Dong, H.; Li, J.; Huang, L.; Chen, X.; Li, D.; Wang, T.; Hu, C.; Xu, J.; Zhang, C.; Zen, K. Serum MicroRNA profiles serve as novel biomarkers for the diagnosis of Alzheimer's disease. Dis. Markers 2015, 2015, 625-659. [CrossRef] [PubMed]

19. Duits, F.H.; Hernandez-Guillamon, M.; Montaner, J.; Goos, J.D.C.; Montañola, A.; Wattjes, M.P.; Barkhof, F.; Scheltens, P.; Teunissen, C.E.; Van Der Flier, W.M. Matrix Metalloproteinases in Alzheimer's Disease and Concurrent Cerebral Microbleeds. J. Alzheimer's Dis. 2015, 48, 711-720. [CrossRef] [PubMed]

20. Hatanaka, H.; Hanyu, H.; Fukasawa, R.; Hirao, K.; Shimizu, S.; Kanetaka, H.; Iwamoto, T. Differences in peripheral oxidative stress markers in Alzheimer's disease, vascular dementia and mixed dementia patients. Geriatr. Gerontol. Int. 2015, 15, 53-58. [CrossRef] [PubMed]

21. Hilal, S.; Chai, Y.L.; Ikram, M.K.; Elangovan, S.; Yeow, T.B.; Xin, X.; Chong, J.Y.; Venketasubramanian, N.; Richards, A.M.; Chong, J.P. Markers of cardiac dysfunction in cognitive impairment and dementia. Medicine (Baltimore) 2015, 94, e297. [CrossRef] [PubMed]

22. Teunissen, C.E.; van der Flier, W.M.; Scheltens, P.; Duits, A.; Wijnstok, N.; Nijpels, G.; Dekker, J.M.; Blankenstein, R.M.A.; Heijboer, A.C. Serum leptin is not altered nor related to cognitive decline in Alzheimer's disease. J. Alzheimer's Dis. 2015, 44, 809-813. [CrossRef] [PubMed]

23. Wang, C.; Zhao, J.; Xu, R.; Zhao, J.; Duan, S. Identification of Pivotal Markers in Vascular Dementia Based on Proteomics Data. Dement. Geriatr. Cogn. Disord. 2015, 39, 312-320. [CrossRef]

24. Castellazzi, M.; Trentini, A.; Romani, A.; Valacchi, G.; Bellini, T.; Bonaccorsi, G.; Fainardi, E.; Cavicchio, C.; Passaro, A.; Zuliani, G. Decreased arylesterase activity of paraoxonase-1 (PON-1) might be a common denominator of neuroinflammatory and neurodegenerative diseases. Int. J. Biochem. Cell Biol. 2016, 81, 356-363. [CrossRef] [PubMed]

25. Chen, Z.; Liang, X.; Zhang, C.; Wang, J.; Chen, G.; Zhang, H.; Sun, Z. Correlation of thyroid dysfunction and cognitive impairments induced by subcortical ischemic vascular disease. Brain Behav. 2016, 6, e00452. [CrossRef] [PubMed]

26. Dukic, L.; Simundic, A.-M.; Martinic-Popovic, I.; Kackov, S.; Diamandis, A.; Begcevic, I.; Diamandis, E.P. The role of human kallikrein 6, clusterin and adiponectin as potential blood biomarkers of dementia. Clin. Biochem. 2016, 49, 213-218. [CrossRef] [PubMed]

27. Horvath, I.; Jia, X.; Johansson, P.; Wang, C.; Moskalenko, R.; Steinau, A.; Forsgren, L.; Wågberg, T.; Svensson, J.; Zetterberg, H.; et al. Pro-inflammatory S100A9 Protein as a Robust Biomarker Differentiating Early Stages of Cognitive Impairment in Alzheimer's Disease. ACS Chem. Neurosci. 2016, 20, 34-39. [CrossRef] [PubMed]

28. Kitagawa, K.; Miwa, K.; Okazaki, S.; Sakaguchi, M.; Mochizuki, H. Serum high-molecular-weight adiponectin level and incident dementia in patients with vascular risk factors. Eur. J. Neurol. 2016, 23, 641-647. [CrossRef] [PubMed] 
29. Levada, O.A.; Cherednichenko, N.V.; Trailin, A.V.; Troyan, A.S. Plasma Brain-Derived Neurotrophic Factor as a Biomarker for the Main Types of Mild Neurocognitive Disorders and Treatment Efficacy: A Preliminary Study. Dis. Markers 2016, 2016, 1-7. [CrossRef]

30. Mirza, S.S.; de Bruijn, R.F.; Koudstaal, P.J.; van den Meiracker, A.H.; Franco, O.H.; Hofman, A.; Tiemeier, H.; Ikram, M.A. The N-terminal pro B-type natriuretic peptide, and risk of dementia and cognitive decline: A 10-year follow-up study in the general population. J. Neurol. Neurosurg. Psychiatry 2016, 87, 356-362. [CrossRef]

31. Nilsson, E.D.; Melander, O.; Elmståhl, S.; Lethagen, E.; Minthon, L.; Pihlsgård, M.; Nägga, K. Copeptin, a Marker of Vasopressin, Predicts Vascular Dementia but not Alzheimer's Disease. J. Alzheimer's Dis. 2016, 52, 1047-1053. [CrossRef]

32. Pan, X.; Fei, G.; Lu, J.; Jin, L.; Pan, S.; Chen, Z.; Wang, C.; Sang, S.; Liu, H.; Hu, W. Measurement of Blood Thiamine Metabolites for Alzheimer's Disease Diagnosis. EBio Med. 2015, 3, 155-162. [CrossRef] [PubMed]

33. Bednarska-Makaruk, M.; Graban, A.; Wiśniewska, A.; Łojkowska, W.; Bochyńska, A.; Gugała-Iwaniuk, M.; Sławińska, K.; Ługowska, A.; Ryglewicz, D.; Wehr, H. Association of adiponectin, leptin and resistin with inflammatory markers and obesity in dementia. Biogerontology 2017, 18, 561-580. [CrossRef] [PubMed]

34. Busse, M.; Michler, E.; Von Hoff, F.; Dobrowolny, H.; Hartig, R.; Frodl, T. Alterations in the Peripheral Immune System in Dementia. J. Alzheimer's Dis. 2017, 58, 1-11. [CrossRef] [PubMed]

35. Holm, H.; Nägga, K.; Nilsson, E.D.; Ricci, F.; Cinosi, E.; Melander, O.; Hansson, O.; Bachus, E.; Magnusson, M.; Fedorowski, A. N-Terminal Prosomatostatin and Risk of Vascular Dementia. Cerebrovasc. Dis. 2017, 44, 259-265. [CrossRef] [PubMed]

36. Holm, H.; Nägga, K.; Nilsson, E.D.; Ricci, F.; Melander, O.; Hansson, O.; Bachus, E.; Magnusson, M.; Fedorowski, A. Biomarkers of microvascular endothelial dysfunction predict incident dementia: A population-based prospective study. J. Intern. Med. 2017, 282, 94-101. [CrossRef]

37. Hsu, P.-F.; Pan, W.-H.; Yip, B.-S.; Chen, R.C.-Y.; Cheng, H.-M.; Chuang, S.-Y. C-Reactive Protein Predicts Incidence of Dementia in an Elderly Asian Community Cohort. J. Am. Med Dir. Assoc. 2017, 18, 277.e7-277.e11. [CrossRef]

38. Moretti, R.; Caruso, P.; Dal Ben, M.; Conti, C.; Gazzin, S.; Tiribelli, C. Vitamin D, Homocysteine, and Folate in Subcortical Vascular Dementia and Alzheimer Dementia. Front. Aging Neurosci. 2017, 30, 169. [CrossRef]

39. Prabhakar, P.; Chandra, S.R.; Christopher, R. Circulating microRNAs as potential biomarkers for the identification of vascular dementia due to cerebral small vessel disease. Age Ageing 2017, 46, 861-864. [CrossRef]

40. Quinlan, P.; Horvath, A.; Nordlund, A.; Wallin, A.; Svensson, J. Low serum insulin-like growth factor-I (IGF-I) level is associated with increased risk of vascular dementia. Psychoneuroendocrinology 2017, 86, 169-175. [CrossRef]

41. Suridjan, I.; Herrmann, N.; Adibfar, A.; Saleem, M.; Andreazza, A.; Oh, P.I.; Lanctôt, K.L.; Naranjo, I.C. Lipid Peroxidation Markers in Coronary Artery Disease Patients with Possible Vascular Mild Cognitive Impairment. J. Alzheimer's Dis. 2017, 58, 885-896. [CrossRef]

42. Tang, S.-C.; Yang, K.-C.; Hu, C.-J.; Chiou, H.-Y.; Wu, C.C.; Jeng, J.-S. Elevated Plasma Level of Soluble Form of RAGE in Ischemic Stroke Patients with Dementia. Neuromol. Med. 2017, 19, 579-583. [CrossRef] [PubMed]

43. Vishnu, V.Y.; Modi, M.; Garg, V.K.; Mohanty, M.; Goyal, M.K.; Lal, V.; Mittal, B.R.; Prabhakar, S. Role of inflammatory and hemostatic biomarkers in Alzheimer's and vascular dementia-A pilot study from a tertiary centre in Northern India. Asian J. Psychiatry 2017, 29, 59-62. [CrossRef] [PubMed]

44. Wang, R.; Chen, Z.; Fu, Y.; Wei, X.; Liao, J.; Liu, X.; He, B.; Xu, Y.; Zou, J.; Yang, X.; et al. Plasma Cystatin C and High-Density Lipoprotein Are Important Biomarkers of Alzheimer's Disease and Vascular Dementia: A Cross-Sectional Study. Front. Aging Neurosci. 2017, 7, 26. [CrossRef] [PubMed]

45. Yang, R.; Fu, S.; Zhao, L.; Zhen, B.; Ye, L.; Niu, X.; Li, X.; Zhang, P.; Bai, J. Quantitation of circulating GDF-11 and $\beta 2-\mathrm{MG}$ in aged patients with age-related impairment in cognitive function. Clin. Sci. (Lond.) 2017, 131, 1895-1904. [CrossRef] [PubMed]

46. Brombo, G.; Bonetti, F.; Ortolani, B.; Morieri, M.L.; Bosi, C.; Passaro, A.; Vigna, G.B.; Borgna, C.; Arcidicono, M.V.; Tisato, V.; et al. Lower Plasma Klotho Concentrations Are Associated with Vascular Dementia but Not Late-Onset Alzheimer's Disease. Gerontology 2018, 64, 414-421. [CrossRef]

47. Latourte, A.; Soumaré, A.; Bardin, T.; Perez-Ruiz, F.; Debette, S.; Richette, P. Uric acid and incident dementia over 12 years of follow-up: A population-based cohort study. Ann. Rheum. Dis. 2018, 77, 328-335. [CrossRef] 
48. Lauriola, M.; Paroni, G.; Ciccone, F.; Onofrio, G.D.; Cascavilla, L.; Paris, F.; Gravina, C.; Urbano, M.; Seripa, D.; Greco, A. Erythrocyte Associated Amyloid- $\beta$ as Potential Biomarker to Diagnose Dementia. Curr. Alzheimer Res. 2018, 15, 381-385. [CrossRef]

49. Shang, J.; Yamashita, T.; Fukui, Y.; Song, D.; Li, X.; Zhai, Y.; Nakano, Y.; Morihara, R.; Hishikawa, N.; Ohta, Y.; et al. Different Associations of Plasma Biomarkers in Alzheimer's Disease, Mild Cognitive Impairment, Vascular Dementia, and Ischemic Stroke. J. Clin. Neurol. 2018, 14, 29-34. [CrossRef]

50. Staszewski, J.; Piusińska-Macoch, R.; Brodacki, B.; Skrobowska, E.; Stępień, A. IL-6, PF-4, sCD40 L, and homocysteine are associated with the radiological progression of cerebral small-vessel disease: A 2-year follow-up study. Clin. Interv. Aging 2018, 13, 1135-1141. [CrossRef]

51. Yang, T.T.; Liu, C.G.; Gao, S.C.; Zhang, Y.; Wang, P.C. The Serum Exosome Derived MicroRNA-135a, -193b, and -384 Were Potential Alzheimer's Disease Biomarkers. Biomed. Environ. Sci. 2018, 31, 87-96.

52. Staszewski, J.; Skrobowska, E.; Piusińska-Macoch, R.; Brodacki, B.; Stępień, A. IL-1 $\alpha$ and IL-6 predict vascular events or death in patients with cerebral small vessel disease-Data from the SHEF-CSVD study. Adv. Med. Sci. 2019, 64, 258-266. [CrossRef] [PubMed]

53. Busse, S.; Busse, M.; Brix, B.; Probst, C.; Genz, A.; Bogerts, B.; Stoecker, W.; Steiner, J. Seroprevalence of n-methyl-d-aspartate glutamate receptor (NMDA-R) autoantibodies in aging subjects without neuropsychiatric disorders and in dementia patients. Eur. Arch. Psychiatry Clin. Neurosci. 2014, 264, 545-550. [CrossRef] [PubMed]

54. Herbert, M.K.; Aerts, M.B.; Kuiperij, H.B.; Claassen, J.A.H.R.; Spies, P.E.; Esselink, R.A.J.; Bloem, B.R.; Verbeek, M.M. Addition of MHPG to Alzheimer's disease biomarkers improves differentiation of dementia with Lewy bodies from Alzheimer's disease but not other dementias. Alzheimer's Dement. 2014, 10, 448-455.e2. [CrossRef] [PubMed]

55. Hermann, P.; Romero, C.; Schmidt, C.; Reis, C.; Zerr, I. CSF biomarkers and neuropsychological profiles in patients with cerebral small-vessel disease. PLoS ONE 2014, 9, e105000. [CrossRef] [PubMed]

56. Skillbäck, T.; Farahmand, B.; Bartlett, J.W.; Rosén, C.; Mattsson, N.; Nägga, K.; Kilander, L.; Religa, D.; Wimo, A.; Winblad, B.; et al. CSF neurofilament light differs in neurodegenerative diseases and predicts severity and survival. Neurology 2014, 83, 1945-1953.

57. Ewers, M.; Mattsson, N.; Minthon, L.; Molinuevo, J.L.; Antonell, A.; Popp, J.; Jessen, F.; Herukka, S.K.; Soininen, H.; Maetzler, W. CSF biomarkers for the differential diagnosis of Alzheimer's disease: A large-scale international multicenter study. Alzheimer's Dement. 2015, 11, 1306-1315. [CrossRef]

58. Liguori, C.; Stefani, A.; Sancesario, G.; Sancesario, G.M.; Marciani, M.G.; Pierantozzi, M. CSF lactate levels, $\tau$ proteins, cognitive decline: A dynamic relationship in Alzheimer's disease. J. Neurol. Neurosurg. Psychiatry 2015, 86, 655-659. [CrossRef]

59. Rosenberg, G.A.; Prestopnik, J.; Adair, J.C.; Huisa, B.N.; Knoefel, J.; Caprihan, A.; Gasparovic, C.; Thompson, J.; Erhardt, E.B.; Schrader, R. Validation of biomarkers in subcortical ischaemic vascular disease of the Binswanger type: Approach to targeted treatment trials. J. Neurol. Neurosurg. Psychiatry 2015, 86, 1324-1330. [CrossRef]

60. Skillbäck, T.; Farahmand, B.Y.; Rosén, C.; Mattsson, N.; Nägga, K.; Kilander, L.; Religa, D.; Wimo, A.; Winblad, B.; Schott, J.M.; et al. Cerebrospinal fluid tau and amyloid- $\beta 1-42$ in patients with dementia. Brain 2015, 138, 2716-2731. [CrossRef]

61. Struyfs, H.; Van Broeck, B.; Timmers, M.; Fransen, E.; Sleegers, K.; Van Broeckhoven, C.; de Deyn, P.P.; Streffer, J.R.; Mercken, M.; Engelborghs, S. Diagnostic Accuracy of Cerebrospinal Fluid Amyloid- $\beta$ Isoforms for Early and Differential Dementia Diagnosis. J. Alzheimer's Dis. 2015, 45, 813-822. [CrossRef]

62. Skillbäck, T.; Delsing, L.; Synnergren, J.; Mattsson, N.; Janelidze, S.; Nägga, K.; Kilander, L.; Hicks, R.; Wimo, A.; Winblad, B.; et al. CSF/serum albumin ratio in dementias: A cross-sectional study on 1861 patients. Neurobiol. Aging 2017, 59, 1-9. [CrossRef] [PubMed]

63. Kiđemet-Piskač, S.; Leko, M.B.; Blažeković, A.; Horvat, L.L.; Klepac, N.; Sonicki, Z.; Kolenc, D.; Hof, P.R.; Boban, M.; Mimica, N.; et al. Evaluation of cerebrospinal fluid phosphorylated tau231 as a biomarker in the differential diagnosis of Alzheimer's disease and vascular dementia. CNS Neurosci. Ther. 2018, 24, 734-740. [CrossRef] [PubMed]

64. Barry Erhardt, E.; Pesko, J.C.; Prestopnik, J.; Thompson, J.; Caprihan, A.; Rosenberg, G.A. Biomarkers identify the Binswanger type of vascular cognitive impairment. J. Cereb. Blood Flow Metab. 2018, 7, 271678X18762655. [CrossRef] [PubMed] 
65. Chakraborty, A.; Chatterjee, M.; Twaalfhoven, H.; Del Campo Milan, M.; Teunissen, C.E.; Scheltens, P.; Fontijn, R.D.; van Der Flier, W.M.; de Vries, H.E. Vascular Endothelial Growth Factor remains unchanged in cerebrospinal fluid of patients with Alzheimer's disease and vascular dementia. Alzheimer's Res. Ther. 2018, 10, 58. [CrossRef] [PubMed]

66. Skrobot, O.A.; Attems, J.; Esiri, M.; Hortobágyi, T.; Ironside, J.W.; Kalaria, R.N.; Lammie, G.A.; Mann, D.; Neal, J.; Ben-Shlomo, Y.; et al. Vascular cognitive impairment neuropathology guidelines (VCING): The contribution of cerebrovascular pathology to cognitive impairment. Brain 2016, 139, 2957-2969. [CrossRef] [PubMed]

67. Wallin, A.; Román, G.C.; Esiri, M.; Kettunen, P.; Svensson, J.; Paraskevas, G.P.; Kapaki, E. Update on Vascular Cognitive Impairment Associated with Subcortical Small-Vessel Disease. J. Alzheimer's Dis. 2018, 62, 1417-1441. [CrossRef] [PubMed]

68. Wardlaw, J.M.; Smith, C.; Dichgans, M. Mechanisms of sporadic cerebral small vessel disease: Insights from neuroimaging. Lancet Neurol. 2013, 12, 483-497. [CrossRef]

69. Ramirez, J.; Berezuk, C.; McNeely, A.A.; Gao, F.; McLaurin, J.; Black, S.E. Imaging the Perivascular Space as a Potential Biomarker of Neurovascular and Neurodegenerative Diseases. Cell. Mol. Neurobiol. 2016, 36, 289-299. [CrossRef] [PubMed]

70. Pantoni, L. Cerebral small vessel disease: From pathogenesis and clinical characteristics to therapeutic challenges. Lancet Neurol. 2010, 9, 689-701. [CrossRef]

71. Joutel, A.; Corpechot, C.; Ducros, A.; Vahedi, K.; Chabriat, H.; Mouton, P.; Alamowitch, S.; Domenga, V.; Cécillion, M.; Maréchal, E.; et al. Notch3 mutations in CADASIL, a hereditary adult-onset condition causing stroke and dementia. Nature 1996, 383, 707-710. [CrossRef]

72. Corlobé, A.; Verdura, E.; Hervé, D.; Scharrer, E.; Amador, M.D.M.; Guyant-Maréchal, L.; Philippi, A.; Bergametti, F.; Gazal, S.; Prieto-Morin, C.; et al. Heterozygous HTRA1 mutations are associated with autosomal dominant cerebral small vessel disease. Brain 2015, 138, 2347-2358.

73. Bugiani, M.; Waisfisz, Q.; Kevelam, S.H.; Bakels, H.S.; Groote, C.C.-D.; Niessen, H.W.M.; Abbink, T.E.M.; Oberstein, S.A.M.J.L.; Van Der Knaap, M.S. Cathepsin A-related arteriopathy with strokes and leukoencephalopathy (CARASAL). Neurology 2016, 87, 1777-1786. [CrossRef] [PubMed]

74. Lynch, D.S.; Jaunmuktane, Z.; Sheerin, U.-M.; Phadke, R.; Brandner, S.; Milonas, I.; Dean, A.; Bajaj, N.; McNicholas, N.; Costello, D.; et al. Hereditary leukoencephalopathy with axonal spheroids: A spectrum of phenotypes from CNS vasculitis to parkinsonism in an adult onset leukodystrophy series. J. Neurol. Neurosurg. Psychiatry 2015, 87, 512-519. [CrossRef] [PubMed]

75. Poggesi, A.; Pasi, M.; Pescini, F.; Pantoni, L.; Inzitari, D. Circulating biologic markers of endothelial dysfunction in cerebral small vessel disease: A review. J. Cereb. Blood Flow Metab. 2016, 36, 72-94. [CrossRef] [PubMed]

76. Mansour, A.; Niizuma, K.; Rashad, S.; Sumiyoshi, A.; Ryoke, R.; Endo, H.; Sato, K.; Kawashima, R.; Tominaga, T. A refined model of chronic cerebral hypoperfusion resulting in cognitive impairment and a low mortality rate in rats. J. Neurosurg. 2018, 1-11. [CrossRef] [PubMed]

77. Wang, F.; Cao, Y.; Ma, L.; Pei, H.; Rausch, W.D.; Li, H. Dysfunction of Cerebrovascular Endothelial Cells: Prelude to Vascular Dementia. Front. Aging Neurosci. 2018, 10. [CrossRef]

78. Young, V.G.; Halliday, G.; Kril, J. Neuropathologic correlates of white matter hyperintensities. Neurology 2008, 71, 804-811. [CrossRef]

79. Fernando, M.S.; Simpson, J.E.; Matthews, F.; Brayne, C.; Lewis, C.E.; Barber, R.; Kalaria, R.N.; Forster, G.; Esteves, F.; Wharton, S.B.; et al. White matter lesions in an unselected cohort of the elderly: Molecular pathology suggests origin from chronic hypoperfusion injury. Stroke 2006, 37, 1391-1398. [CrossRef]

80. Duncombe, J.; Kitamura, A.; Hase, Y.; Ihara, M.; Kalaria, R.N.; Horsburgh, K. Chronic cerebral hypoperfusion: A key mechanism leading to vascular cognitive impairment and dementia. Closing the translational gap between rodent models and human vascular cognitive impairment and dementia. Clin. Sci. 2017, 131, 2451-2468. [CrossRef]

81. Abbott, N.J.; Patabendige, A.A.; Dolman, D.E.; Yusof, S.R.; Begley, D.J. Structure and function of the blood-brain barrier. Neurobiol. Dis. 2010, 37, 13-25. [CrossRef]

82. Ueno, M.; Chiba, Y.; Matsumoto, K.; Murakami, R.; Fujihara, R.; Kawauchi, M.; Miyanaka, H.; Nakagawa, T. Blood-brain barrier damage in vascular dementia. Neuropathology 2016, 36, 115-124. [CrossRef] [PubMed] 
83. Farrall, A.J.; Wardlaw, J.M. Blood-brain barrier: Ageing and microvascular disease-systematic review and meta-analysis. Neurobiol. Aging 2009, 30, 337-352. [CrossRef] [PubMed]

84. Amor, S.; Peferoen, L.A.N.; Vogel, D.Y.S.; Breur, M.; Valk, P.; Baker, D.; Noort, J.M.; Van Der Valk, P.; van Noort, J.M. Inflammation in neurodegenerative diseases-An update. Immunology 2014, 142, 151-166. [CrossRef] [PubMed]

85. Rouhl, R.P.; Damoiseaux, J.G.; Lodder, J.; Theunissen, R.O.; Knottnerus, I.L.; Staals, J.; Henskens, L.H.; Kroon, A.A.; De Leeuw, P.W.; Tervaert, J.W.C.; et al. Vascular inflammation in cerebral small vessel disease. Neurobiol. Aging 2012, 33, 1800-1806. [CrossRef] [PubMed]

86. Venkat, P.; Chopp, M.; Chen, J. Models and mechanisms of vascular dementia. Exp. Neurol. 2015, 272, 97-108. [CrossRef] [PubMed]

87. Vijayan, M.; Kumar, S.; Bhatti, J.S.; Reddy, P.H. Molecular Links and Biomarkers of Stroke, Vascular Dementia, and Alzheimer's Disease. Prog. Mol. Biol. Transl. Sci. 2017, 146, 95-126.

88. Sullivan, G.W.; Sarembock, I.J.; Linden, J. The role of inflammation in vascular diseases. J. Leukoc. Biol. 2000, 67, 591-602. [CrossRef]

89. Gu, Y.; Gutierrez, J.; Meier, I.B.; Guzman, V.A.; Manly, J.J.; Schupf, N.; Brickman, A.M.; Mayeux, R. Circulating inflammatory biomarkers are related to cerebrovascular disease in older adults. Neurol. - Neuroimmunol. Neuroinflamm. 2018, 6, e521. [CrossRef]

90. Belkhelfa, M.; Beder, N.; Mouhoub, D.; Amri, M.; Hayet, R.; Tighilt, N.; Bakheti, S.; Laimouche, S.; Azzouz, D.; Belhadj, R.; et al. The involvement of neuroinflammation and necroptosis in the hippocampus during vascular dementia. J. Neuroimmunol. 2018, 15, 48-57. [CrossRef]

91. Nagai, K.; Kozaki, K.; Sonohara, K.; Akishita, M.; Toba, K. Relationship between interleukin-6 and cerebral deep white matter and periventricular hyperintensity in elderly women. Geriatr. Gerontol. Int. 2011, 11, 328-332. [CrossRef]

92. Hilal, S.; Ikram, M.A.; Verbeek, M.M.; Franco, O.H.; Stoops, E.; Vanderstichele, H.; Niessen, W.J.; Vernooij, M.W. C-Reactive Protein, Plasma Amyloid- $\beta$ Levels, and Their Interaction with Magnetic Resonance Imaging Markers. Stroke 2018, 49, 2692-2698. [CrossRef] [PubMed]

93. Walker, K.A.; Power, M.C.; Hoogeveen, R.C.; Folsom, A.R.; Ballantyne, C.M.; Knopman, D.S.; Windham, B.G.; Selvin, E.; Jack, C.R., Jr.; Gottesman, R.F. Midlife systemic inflammation, late-life white matter integrity, and cerebral small vessel disease: The atherosclerosis risk in communities study. Stroke 2017, 48, 3196-3202. [CrossRef] [PubMed]

94. Satizabal, C.; Zhu, Y.C.; Mazoyer, B.; Dufouil, C.; Tzourio, C. Circulating IL-6 and CRP are associated with MRI findings in the elderly: The 3C-Dijon Study. Neurology 2012, 78, 720-727. [CrossRef] [PubMed]

95. Bettcher, B.M.; Yaffe, K.; Boudreau, R.M.; Neuhaus, J.; Aizenstein, H.; Ding, J.; Kritchevsky, S.B.; Launer, L.J.; Liu, Y.; Satterfield, S.; et al. Declines in inflammation predict greater white matter microstructure in older adults. Neurobiol. Aging 2015, 36, 948-954. [CrossRef] [PubMed]

96. Mitaki, S.; Nagai, A.; Oguro, H.; Yamaguchi, S. C-Reactive Protein Levels are Associated with Cerebral Small Vessel-Related Lesions. Acta Neurol. Scand. 2016, 133, 68-74. [CrossRef] [PubMed]

97. Han, J.H.; Wong, K.S.; Wang, Y.Y.; Fu, J.H.; Ding, D.; Hong, Z.; Wong, K.S.L. Plasma level of sICAM-1 is associated with the extent of white matter lesion among asymptomatic elderly subjects. Clin. Neurol. Neurosurg. 2009, 111, 847-851. [CrossRef] [PubMed]

98. Markus, H.S.; Hunt, B.; Palmer, K.; Enzinger, C.; Schmidt, H.; Schmidt, R. Markers of endothelial and hemostatic activation and progression of cerebral white matter hyperintensities: Longitudinal results of the Austrian Stroke Prevention Study. Stroke 2005, 36, 1410-1414. [CrossRef] [PubMed]

99. Jalal, F.Y.; Yang, Y.; Thompson, J.; Lopez, A.C.; Rosenberg, G.A. Myelin loss associated with neuroinflammation in hypertensive rats. Stroke 2012, 43, 1115-1122. [CrossRef] [PubMed]

100. Weekman, E.M.; Wilcock, D.M. Matrix metalloproteinase in blood-brain barrier breakdown in dementia. J. Alzheimer's Dis. 2016, 49, 893-903. [CrossRef]

101. Bjerke, M.; Zetterberg, H.; Edman, Å.; Blennow, K.; Wallin, A.; Andreasson, U. Cerebrospinal Fluid Matrix Metalloproteinases and Tissue Inhibitor of Metalloproteinases in Combination with Subcortical and Cortical Biomarkers in Vascular Dementia and Alzheimer's Disease. J. Alzheimer's Dis. 2011, 27, 665-676. [CrossRef]

102. Candelario-Jalil, E.; Thompson, J.; Taheri, S.; Grossetete, M.; Adair, J.C.; Edmonds, E.; Prestopnik, J.; Wills, J.; Rosenberg, G.A.; Adair, J. Matrix Metalloproteinases are Associated with Increased Blood-Brain Barrier Opening in Vascular Cognitive Impairment. Stroke 2011, 42, 1345-1350. [CrossRef] [PubMed] 
103. Lüscher, C.; Malenka, R.C. NMDA Receptor-Dependent Long-Term Potentiation and Long-Term Depression (LTP/LTD). Cold Spring Harb. Perspect. Biol. 2012, 4, a005710.

104. Ihara, M.; Polvikoski, T.M.; Hall, R.; Slade, J.Y.; Perry, R.H.; Oakley, A.E.; Englund, E.; O’Brien, J.T.; Ince, P.G.; Kalaria, R.N. Quantification of myelin loss in frontal lobe white matter in vascular dementia, Alzheimer's disease, and dementia with Lewy bodies. Acta Neuropathol. 2010, 119, 579-589. [CrossRef] [PubMed]

105. Barker, R.; Wellington, D.; Esiri, M.M.; Love, S. Assessing white matter ischemic damage in dementia patients by measurement of myelin proteins. J. Cereb. Blood Flow Metab. 2013, 33, 1050-1057. [CrossRef] [PubMed]

106. Llorens, F.; Schmitz, M.; Knipper, T.; Schmidt, C.; Lange, P.; Fischer, A.; Hermann, P.; Zerr, I. Cerebrospinal Fluid Biomarkers of Alzheimer's Disease Show Different but Partially Overlapping Profile Compared to Vascular Dementia. Front. Aging Neurosci. 2017, 9, 289. [CrossRef] [PubMed]

107. Duering, M.; Konieczny, M.J.; Tiedt, S.; Baykara, E.; Tuladhar, A.M.; Van Leijsen, E.; Lyrer, P.; Engelter, S.T.; Gesierich, B.; Achmüller, M.; et al. Serum Neurofilament Light Chain Levels Are Related to Small Vessel Disease Burden. J. Stroke 2018, 20, 228-238. [CrossRef] [PubMed]

108. Gravesteijn, G.; Rutten, J.W.; Verberk, I.M.W.; Böhringer, S.; Liem, M.K.; van der Grond, J.; Aartsma-Rus, A.; Teunissen, C.E.; Oberstein, S.A.J.L. Serum Neurofilament light correlates with CADASIL disease severity and survival. Ann. Clin. Transl. Neurol. 2018, 6, 46-56. [CrossRef]

109. Rothwell, P.M.; Howard, S.C.; Power, D.A.; Gutnikov, S.A.; Algra, A.; Van Gijn, J.; Clark, T.G.; Murphy, M.F.; Warlow, C.P. Fibrinogen Concentration and Risk of Ischemic Stroke and Acute Coronary Events in 5113 Patients with Transient Ischemic Attack and Minor Ischemic Stroke. Stroke 2004, 35, 2300-2305. [CrossRef]

110. Di Napoli, M.; Papa, F.; Bocola, V. Prognostic influence of increased C-reactive protein and fibrinogen levels in ischemic stroke. Stroke 2001, 32, 133-138. [CrossRef]

111. Xu, G.; Zhang, H.; Zhang, S.; Fan, X.; Liu, X. Plasma fibrinogen is associated with cognitive decline and risk for dementia in patients with mild cognitive impairment. Int. J. Clin. Pract. 2008, 62, 1070-1075. [CrossRef]

112. Fitzpatrick, A.L.; Irizarry, M.C.; Cushman, M.; Jenny, N.S.; Chi, G.C.; Koro, C. Lipoprotein-associated phospholipase A2 and Risk of Dementia in the Cardiovascular Health Study. Atherosclerosis 2014, 235, 384-391. [CrossRef] [PubMed]

113. Hunt, B.J.; Parmar, K.; Bamford, J.M.; Briley, D.; Brown, M.M.; Thomas, D.J.; Markus, H.S.; Hassan, A.; O'Sullivan, M. Markers of endothelial dysfunction in lacunar infarction and ischaemic leukoaraiosis. Brain 2003, 126, 424-432.

114. Kearney-Schwartz, A.; Rossignol, P.; Bracard, S.; Felblinger, J.; Fay, R.; Boivin, J.-M.; Lecompte, T.; Lacolley, P.; Benetos, A.; Zannad, F. Vascular Structure and Function Is Correlated to Cognitive Performance and White Matter Hyperintensities in Older Hypertensive Patients with Subjective Memory Complaints. Stroke 2009, 40, 1229-1236. [CrossRef] [PubMed]

115. Nagai, M.; Hoshide, S.; Kario, K. Association of Prothrombotic Status with Markers of Cerebral Small Vessel Disease in Elderly Hypertensive Patients. Am. J. Hypertens. 2012, 25, 1088-1094. [CrossRef] [PubMed]

116. Pescini, F.; Donnini, I.; Cesari, F.; Nannucci, S.; Valenti, R.; Rinnoci, V.; Poggesi, A.; Gori, A.M.; Giusti, B.; Rogolino, A.; et al. Circulating Biomarkers in Cerebral Autosomal Dominant Arteriopathy with Subcortical Infarcts and Leukoencephalopathy Patients. J. Stroke Cerebrovasc. Dis. 2017, 26, 823-833. [CrossRef] [PubMed]

117. Bevan, S.; Barrick, T.; Rich, P.; Markus, H.S.; Singhal, S. The influence of genetic and cardiovascular risk factors on the CADASIL phenotype. Brain 2004, 127, 2031-2038.

118. Pescini, F.; Cesari, F.; Giusti, B.; Sarti, C.; Zicari, E.; Bianchi, S.; Dotti, M.T.; Federico, A.; Balestrino, M.; Enrico, A.; et al. Bone marrow-derived progenitor cells in cerebral autosomal dominant arteriopathy with subcortical infarcts and leukoencephalopathy. Stroke 2010, 41, 218-223. [CrossRef] [PubMed]

119. Zheng, L.L.; Li, J.H.; Wu, J.; Sun, W.J.; Liu, S.; Wang, Z.L.; Zhou, H.; Yang, J.-H.; Qu, L.-H. deepBase v2.0: Identification, expression, evolution and function of small RNAs, IncRNAs and circular RNAs from deep-sequencing data. Nucleic Acids Res. 2016, 44, D196-D202. [CrossRef]

120. Vemuganti, R. The MicroRNAs and Stroke: No Need to be Coded to be Counted. Transl. Stroke Res. 2010, 1, 158-160. [CrossRef]

121. Li, J.; Liu, Y.; Xin, X.; Kim, T.S.; Cabeza, E.A.; Ren, J.; Nielsen, R.; Wrana, J.L.; Zhang, Z. Evidence for Positive Selection on a Number of MicroRNA Regulatory Interactions during Recent Human Evolution. PLoS Genet. 2012, 8, 1002578. [CrossRef]

122. Weidhaas, J. Using microRNAs to understand cancer biology. Lancet Oncol. 2010, 11, 106-107. [CrossRef] 
123. Li, M.; Zhang, J. Circulating microRNAs. Potential and emerging bio-markers for diagnosis of cardiovascular and cerebrovascular diseases. Biomed. Res. Int. 2015, 2015. [CrossRef]

124. Chen, X.; Ba, Y.; Ma, L.; Cai, X.; Yin, Y.; Wang, K.; Guo, J.; Zhang, Y.; Chen, J.; Guo, X.; et al. Characterization of microRNAs in serum: A novel class of biomarkers for diagnosis of cancer and other diseases. Cell Res. 2008, 18, 997-1006. [CrossRef] [PubMed]

125. Sano, T.; Reynolds, J.P.; Jimenez-Mateos, E.M.; Matsushima, S.; Taki, W.; Henshall, D.C.; Henshall, D. MicroRNA-34a upregulation during seizure-induced neuronal death. Cell Death Dis. 2012, 3, e287. [CrossRef] [PubMed]

126. Vemuganti, R. All's Well That Transcribes Well: Non-coding RNAs and Post-Stroke Brain Damage. Neurochem. Int. 2013, 63, 438-449. [CrossRef]

127. Ragusa, M.; Bosco, P.; Tamburello, L.; Barbagallo, C.; Condorelli, A.G.; Tornitore, M.; Spada, R.S.; Barbagallo, D.; Scalia, M.; Elia, M.; et al. miRNAs Plasma Profiles in Vascular Dementia: Biomolecular Data and Biomedical Implications. Front. Cell. Neurosci. 2016, 10, 1. [CrossRef]

128. Sørensen, S.S.; Nygaard, A.B.; Christensen, T. miRNA expression profiles in cerebrospinal fluid and blood of patients with Alzheimer's disease and other types of dementia-An exploratory study. Transl. Neurodegener. 2016, 5, 6 .

(C) 2019 by the authors. Licensee MDPI, Basel, Switzerland. This article is an open access article distributed under the terms and conditions of the Creative Commons Attribution (CC BY) license (http://creativecommons.org/licenses/by/4.0/). 